\title{
Stem cell transplantation impairs dendritic cell trafficking and herpesvirus immunity
}

Carol A. Wilke, ${ }^{1}$ Mathew M. Chadwick, ${ }^{1}$ Paul R. Chan, ${ }^{1}$ Bethany B. Moore, ${ }^{1,2}$ and Xiaofeng Zhou ${ }^{1}$

'Division of Pulmonary and Critical Care Medicine, Department of Internal Medicine, University of Michigan Medical School, Ann Arbor, Michigan, USA. ${ }^{2}$ Department of Microbiology and Immunology, University of Michigan, Ann Arbor, Michigan, USA.

Long-term survivors after hematopoietic stem cell transplantation are at high risk of infection, which accounts for one-third of all deaths related to stem cell transplantation. Little is known about the cause of inferior host defense after immune cell reconstitution. Here, we exploited a murine syngeneic BM transplantation (BMT) model of late infection with murine gammaherpesvirus 68 (MHV-68) to determine the role of conventional DC (CDC) trafficking in adaptive immunity in BMT mice. After infection, the expression of chemokine $\mathrm{Cc} / 21$ in the lung is reduced and the migration of CDCs into lung draining lymph nodes (dLNs) is impaired in BMT mice, limiting the opportunity for cDCs to prime Th cells in the dLNs. While CDC subsets are redundant in priming Th1 cells, Notch2 functions in CDC2s are required for priming increased Th17 responses in BMT mice, and CDC1s can lessen this activity. Importantly, Th17 cells can be primed both in the lungs and dLNs, allowing for increased Th17 responses without optimum CDC trafficking in BMT mice. Taken together, impaired cDC trafficking in BMT mice reduces protective Th1 responses and allows increased pathogenic Th17 responses. Thus, we have revealed a previously unknown mechanism for BMT procedures to cause long-term inferior immune responses to herpes viral infection.

Conflict of interest: The authors have declared that no conflict of interest exists.

Copyright: (c) 2019 American Society for Clinical Investigation

Submitted: May 13, 2019

Accepted: August 21, 2019

Published: August 27, 2019.

Reference information: /CI Insight. 2019;4(18):e130210. https://doi. org/10.1172/jii.insight.130210

\section{Introduction}

Hematopoietic stem cell transplantation (SCT) is a curative therapy for many malignant, autoimmune, and inherited disorders; however, infectious complications are a leading cause of morbidity and nonrelapse mortality after SCT $(1,2)$. SCT conditioning disrupts the natural barriers of the epithelium and mucous membranes and destroys or severely weakens the host immune system (3). The recovery of T lymphocyte counts, especially $\mathrm{CD}^{+} \mathrm{T}$ cells, predicts better survival and reduced risk of infections $(1,4-8)$. Regeneration of naive $\mathrm{CD}^{+} \mathrm{T}$ cells occurs from 4-24 months in patients younger than 45 years (9). However, patients who survive more than 2 years after allogeneic SCT are still at considerably higher risk of infection than the age-matched general population (10-12), and infectious diseases account for one-third of all deaths (13). Infections are less common in autologous SCT recipients; nevertheless, infectious diseases contribute to one-third of late nonrelapse mortality among this group of patients (14), suggesting that even in the absence of chronic graft-versus-host disease (cGVHD) and immunosuppressive therapy, autologous SCT recipients still suffer from long-term immune dysfunction.

The inferior immune defense against infections after full reconstitution of $\mathrm{T}$ lymphocytes is not well understood but may be due to the reconstitution and activation of $\mathrm{T}$ cells in a SCT-altered microenvironment that differs from normal conditions (9). A conditioning-induced proinflammatory cytokine storm that lasts for weeks after SCT (15-17) shapes the antigen-specific T lymphocyte repertoire and Th cell phenotypes (i.e., Treg, Th1, Th2, and Th17; ref. 18). DCs, a potent population of professional antigen-presenting cells (APCs) that activate and define $\mathrm{T}$ cell responses, reconstitute rapidly after transplant but with prolonged skewing of cell subsets (16). Furthermore, SCT conditioning damages the fibroblastic reticular cells and their expression of CCL21 in secondary lymphatic organs (19-21). CCL21 is essential for lymph nodes (LNs) to recruit naive T cells (22) and DCs (23). We recently demonstrated that cytokine production of APCs is dysregulated in response to infection with herpesvirus and causes a Th17 phenotype in a mouse syngeneic BM transplant (BMT) model (24). Clearly, the unique microenvironmental conditions after SCT play an important role in shaping recipient immune reconstitution. 
Reactivations of latent human herpesviruses (HHVs) are common during the early postengraftment phase and throughout late postengraftment $(1,25,26)$. Cytomegalovirus (CMV), HHV-6, Epstein-Barr virus (EBV), and varicella zoster virus (VZV) are among the most frequently reactivated viruses after SCT. HHV infections after SCT can lead to life-threatening diseases such as pneumonitis, encephalitis, or posttransplant lymphoproliferative disease (25). In addition, we and others have recently revealed a strong association between reactivations/infections with herpes or respiratory viruses and the risk of high mortality noninfectious pulmonary complications after SCT, such as idiopathic pneumonia syndrome and bronchiolitis obliterans syndrome (27-30).

To better understand the mechanisms underlying the pathogenesis of herpes viral infections after SCT, we modeled the infection by inoculating murine gammaherpesvirus 68 (MHV-68), a mouse homo$\log$ of EBV and Kaposi's sarcoma-associated herpesvirus, into syngeneic BMT recipients (BMT mice) i.n. 5 weeks after BMT. Note that innate immune cell numbers are restored at 3 weeks after BMT (31) and $\mathrm{T}$ lymphocyte regeneration in the thymus (19) and reconstitution in the lung and spleen are complete at 5 weeks (32) in syngeneic BMT mice. This model allows us to study transplant-related alterations to immune cell function without the added complication of allo-immune responses. However, even after full immune cell reconstitution, BMT mice can still experience impaired innate and adaptive immunity to bacterial or viral infection (reviewed in ref. 33). BMT mice developed severe pneumonitis and lung fibrosis 3 weeks after infection with MHV-68, whereas infected non-BMT mice showed no pathology $(24,34,35)$. This lung pathology was driven by pathogenic Th17 responses with weakened protective Th1 responses (24). APCs isolated from the lungs of BMT mice not only produce increased levels of pro-Th17 cytokines, such as IL-6, TGF- $\beta$, and IL-23, but surprisingly also produce high levels of the pro-Th1 cytokine IL-12. This is in contrast to the suppressed anti-viral Th1 responses in vivo (24). Lung-resident conventional DCs (cDCs) are the most efficient APCs in the lung in sampling air-borne and blood-borne pathogens (36). After taking up and processing antigens, $\mathrm{cDCs}$ are thought to migrate into the mediastinal draining lymph node $(\mathrm{dLN})$ to present antigens to and prime naive $\mathrm{T}$ cells (37). There are 2 major lineages of cDCs in the lung - i.e., type 1 cDCs (cDC1s) expressing CD8 $\alpha, C D 24$, XCR1, and CD103, and cDC2s expressing CD4, Sirp $\alpha$, and CD11b $(38,39)$. cDC1s are responsible for cross-presenting antigens to $\mathrm{CD}^{+} \mathrm{T}$ cells for antiviral immunity, while $\mathrm{cDC} 2 \mathrm{~s}$ have been shown to support Th2 and Th17 cell responses (39).

Here, we report underlying mechanisms for the insufficient protective, yet pathogenic immune response to infection with gammaherpesvirus in syngeneic BMT mice after full immune reconstitution. We found that, although lung cDCs of BMT mice are potent in inducing Th1 responses ex vivo, they are unable to induce strong Th1 responses in vivo due to their impaired migration into lung dLNs to prime T cells after infection. Furthermore, a subset of Notch2 functionally dependent cDC2s are solely responsible for priming Th17 cells, while cDC1s suppress excessive Th17 responses. Our data also suggest that impaired migration of cDC2s allows for increased Th17 responses in BMT mice, as cDC2s can locally prime Th17 cells in the lungs without entering into the dLNs. Thus, our study sheds light on understanding the vulnerability of SCT recipients to infections even after immune reconstitution.

\section{Results}

APCs from lungs of BMT mice are potent in stimulating both Th1 and Th17 responses. We previously reported, and confirm again here, that BMT mice display reduced protective Th1 responses and increased pathogenic Th17 responses in the lungs 7 days after i.n. inoculation with $5 \times 10^{4}$ pfu MHV-68 (Figure 1A) (24). The BMT mice develop severe pneumonitis and lung fibrosis 3 weeks after infection, and IL-17A is essential for the development of the lung pathology $(24,34)$. Surprisingly, APCs isolated from BMT mice express higher levels of the pro-Th1 cytokine IL-12p35 than APCs from non-BMT mice, even though the Th1 responses in the BMT mice are decreased in vivo (24).

To determine if APCs from BMT mice are capable of stimulating potent Th1 as well as Th17 responses, we isolated $\mathrm{CD} 11 \mathrm{c}^{+} \mathrm{APCs}$ (including macrophages and DCs) and $\mathrm{CD}^{+} \mathrm{T}$ cells from the lungs of infected BMT mice and cocultured them (1:10 ratio) for 4 days. Concentrations of both IFN- $\gamma$ and IL-17A in the supernatant of the cocultures with BMT APCs were significantly higher than that with non-BMT APCs (Figure 1B). Indeed, increased mRNA expression levels of both pro-Th1 and pro-Th17 cytokines (i.e., Il12a, Il6, and Il23a) were found in cocultures with BMT APCs compared with non-BMT APCs (Figure 1C). Thus, APCs from MHV-68-infected BMT mice are capable of stimulating T cells to produce high levels of IFN- $\gamma$ in vitro. 
A

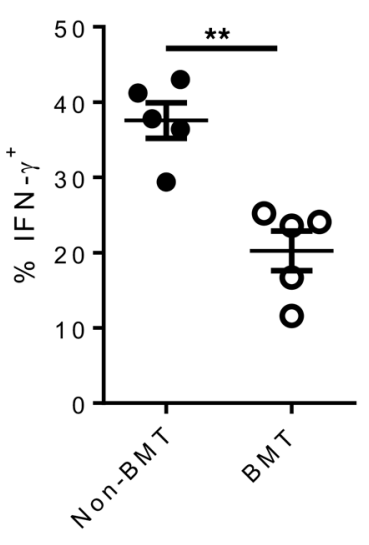

C

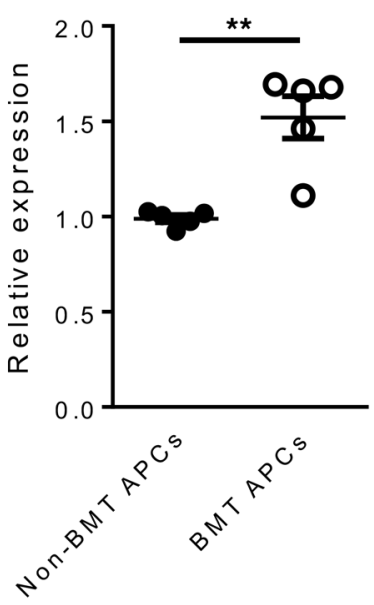

Th17\%

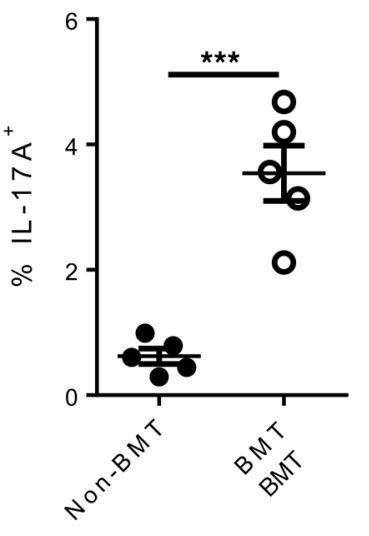

116

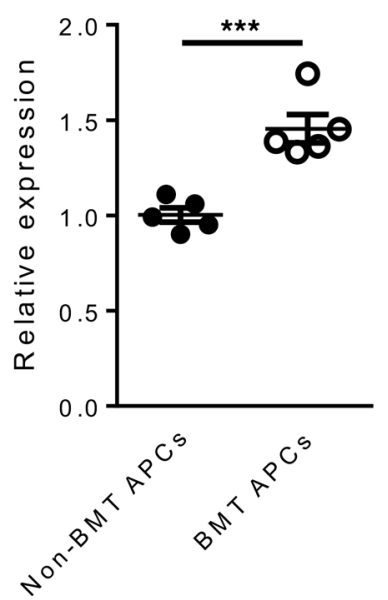

B
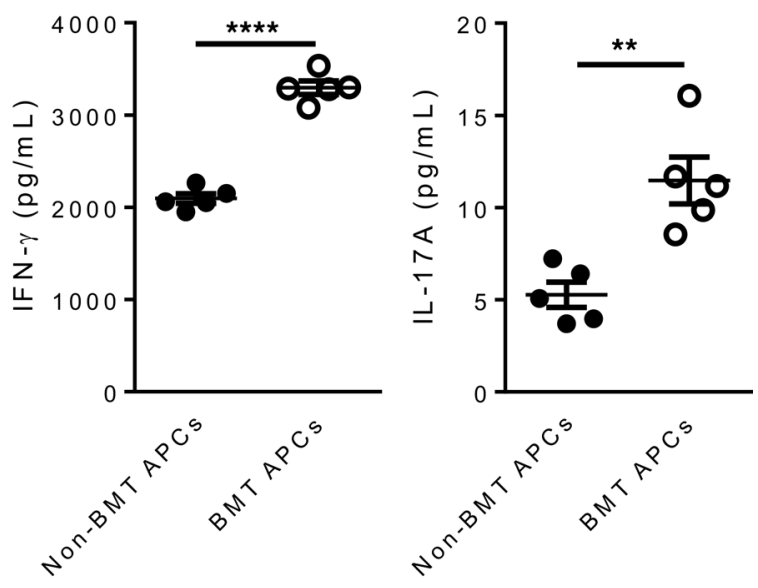

$1 / 23 a$

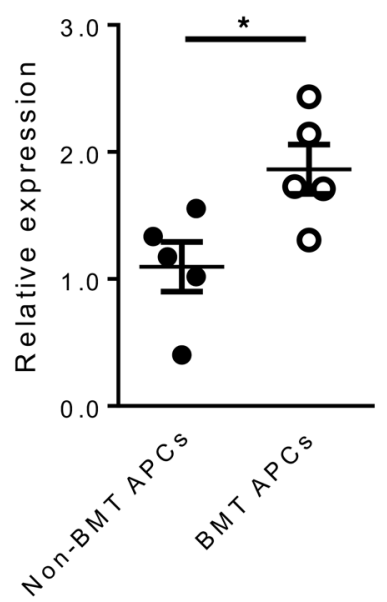

Figure 1. APCs from lungs of BMT mice are potent in priming both Th1 and Th17 responses in vitro. (A) Single-cell suspensions were prepared by collagenase digestion of whole lungs of non-BMT $(n=5)$ or BMT $(n=5)$ mice at $7 \mathrm{dpi}$ with MHV-68. Cells were then stimulated with PMA and ionomycin for 4 hours before antibody staining. Percent of CD4+ $T$ cells (i.e., CD45+CD90.2+CD3+CD4+) that express IFN- $\gamma$ (Th1 cells), and percent of CD4 ${ }^{+}$T cells that express IL-17A (Th17 cells) were determined by flow cytometry. (B and C) APCs enriched by CD11 ${ }^{+}$microbeads and pooled from lung Single-cell suspensions of 5 BMT or non-BMT mice at 3 dpi were cocultured with pooled CD4+ T cells from 10 BMT mouse lungs at $10 \mathrm{dpi}$ at a 1:10 ratio in the presence of $0.125 \mathrm{MOI}$ of MHV- 68 ( $n=5$ each). (B) The concentration of IFN- $\gamma$ or IL-17A in the supernatant of coculture at 4 days was determined by ELISA (mean \pm SEM, $n=5$ ). (C) Cocultured cells were pelleted at 4 days after coculture, and total RNA was isolated by TRIzol. The expression of a pro-Th1 cytokine (IL12A) or pro-Th17 cytokines (IL6 and IL23A) were determined by real-time RT-PCR (mean \pm SEM, $n=5$ ). Symbols represent individual data points from unique mice. ${ }^{*} P<0.05 ;{ }^{* *} P<0.01$; ${ }^{* * *} P<0.001$; ${ }^{* * *} P<0.0001$, Student's $t$ test ( 2 tailed). Similar results were obtained in 2 additional experiments. APCs, antigen-presenting cells; BMT, BM transplantation.

$D C$ migration is impaired in lungs of BMT mice. The discrepant results between the in vitro and in vivo studies suggest that APCs from BMT mice can fully interact with and stimulate CD4 $\mathrm{T}$ cells in vitro, but they may not have optimum interactions with $\mathrm{T}$ cells in vivo. We thus hypothesized that cDCs are impaired in migrating from the lungs to the draining LNs (dLN) in BMT mice to prime naive $\mathrm{T}$ cells. We first enumerated $\mathrm{cDC}$ in lungs based on their high expression of CD11c and MHC class II molecules, and their low levels of Siglec-F and CD64, which are markers for macrophages and monocyte-derived DCs (40-42) (Figure 2A). These cDCs can be further identified as CDC1 and cDC2 cells based on their discrepant expression levels of CD103/CD24 and CD11b (Figure 2A). Migratory DCs (mDCs) moving from the lung to the $\mathrm{dLN}$ can also be classified based on their high expression of MHC class II molecules and high or intermediate levels of CD11c (Figure 2A). In naive mice or mice 2 days post infection (dpi) with MHV-68, similar number of $\mathrm{CDC} 1 \mathrm{~s}$ are present in the lungs of control (non-BMT) and BMT recipients (Figure $2 \mathrm{~B}$ ). There are fewer $\mathrm{CDC} 2 \mathrm{~s}$ in the lungs of non-BMT mice at day 0 , but by $2 \mathrm{dpi}$, numbers are similar to each other (Figure 2B). However, fewer cDC1s or cDC2s remain in the lungs of normal mice compared with BMT recipients at 3 dpi (Figure 2B), suggesting 
that, in normal mice, more cDCs emigrate out of the lungs than in BMT mice during the first 2-3 dpi with MHV-68. In agreement with more cDCs remaining in the lungs in BMT mice, there are significantly fewer migratory $\mathrm{cDC} 1 \mathrm{~s}$ and $\mathrm{cDC} 2 \mathrm{~s}$ in the dLNs in BMT mice at 3 dpi with MHV-68 (Figure 2B).

To track the migration of cDCs from the lung to the dLN, non-BMT or BMT mice at 2 dpi with MHV68 were instilled with $5 \mathrm{mM}$ CFSE via oropharyngeal aspiration to label all the cells in the lungs, and at 18 hours after CFSE instillation, dLNs were harvested to enumerate $\mathrm{CFSE}^{+} \mathrm{mDCs}$ by flow cytometry analysis (Figure 2C). Both the percentages and absolute numbers of $\mathrm{CFSE}^{+} \mathrm{CDC} 1 \mathrm{~s}$ and $\mathrm{cDC} 2 \mathrm{~s}$ were significantly reduced in BMT dLNs compared with the non-BMT mice (Figure 2, C and D). Taken together, our data demonstrate that the migration of lung $\mathrm{CDCs}$ to the $\mathrm{dLN}$ is impaired in BMT mice.

Reduction in Th1 cell differentiation and cellularity in dLNs in BMT mice. To examine whether impaired cDC migration affects subsequent priming of Th cells in lung dLNs, we enumerated and characterized the cells in the dLNs at 7 dpi with MHV-68. Indeed, the absolute numbers of Th1 cells in the dLNs of BMT mice are significantly reduced (Figure 3A), consistent with limited Th1 responses in BMT mice. Notably, although fewer in cell counts, the percentages of Th1 cells within the dLNs are comparable between non-BMT and BMT mice (Figure 3A), suggesting that the $\mathrm{CDCs}$ of BMT mice are potent in priming Th1 responses once reaching the dLNs. While the numbers of Th17 cells are comparable between normal and BMT mice in the dLNs, the Th17 cells of BMT mice make up a larger percentage of the population than those in non-BMT mice (Figure 3B), further confirming a Th17-skewed response. It has been previously reported that both allogeneic and congeneic BMT reduces steady state cellularity in LNs after immune reconstitution and that BMT mice generate poor responses to bacterial and viral infections (19-21). Similarly, we also found that there is about a 3-fold reduction in total cell numbers in BMT lung dLNs at 7 dpi, with significantly fewer mDCs, CD4 ${ }^{+}$Th cells, $\mathrm{CD}^{+} \mathrm{T}$ cells, and B cells in the BMT dLNs (Figure 3C).

BMT downregulates the expression of chemokine Ccl21 in the lung and dLN but does not affect the surface expression of CCR7 on CDCs. CC-chemokine receptor 7 (CCR7) is essential for mature CDCs to sense and follow the gradient of CCL21 toward and into the lumen of lymphatic vessels leading to dLNs (43). Interestingly, the expression of CCR7 on lung $\mathrm{CDC}$ s is similar between non-BMT and BMT mice at 2 dpi when activated lung cDCs are ready to migrate (Figure $4 \mathrm{~A}$ ). There are 3 distinct $C c l 21$ genes in the mouse. $C c l 21 a$ encodes CCL21Ser and is predominantly expressed in lymphoid organs, while Ccl21b and Ccl21c both encode CCL$21 \mathrm{Leu}$ and are predominantly expressed in nonimmune organs, such as the lung (44). CCL19 is the other ligand for CCR7, and it is also predominantly present within the dLNs (45). We found that the expression levels of $C c l 21 a$ and $C c l 21 b / c$ are reduced in the lungs of BMT mice at 3 dpi (Figure 4B), consistent with the observation that cDCs stall in the lungs in BMT mice after infection with MHV-68. We also note that levels of $C c l 21 a$ and $C c l 21 b / c$ transcripts are similar between the dLNs of non-BMT and BMT mice prior to infection and at 3 dpi (Figure 4B); however, both BMT and non-BMT dLNs show reduced transcription of Ccl21 at 7 dpi, but BMT dLNs show greater reduction (Figure 4B). To further determine the cells producing CCL21 in the lungs, we have performed multicolor immunofluorescence staining of lung frozen sections. Labeling of CD90.2 protein identifies lymph vessels in the lung (46). Similar to previous studies (reviewed in ref. 47), we found that CCL21 protein is exclusively expressed in lymphatic endothelial cells in the lung prior to infection (data not shown) and at 3 dpi (Figure 4C). Importantly, the number of CD11 $\mathrm{c}^{+}$cells, presumably cDCs, within or in contact with lymph vessels is reduced in BMT lungs at 3 dpi (Figure 4C). These observations indicate that the impaired $\mathrm{CDC}$ migration in BMT mice is not due to the regulation of CCR7 on the $\mathrm{CDC}$ cell surface, but rather is likely due to insufficient production of CCL21 in the lungs.

Disparate functions of $c D C 1 s$ and $c D C 2 s$ in priming Th17 differentiation. As shown above, the impaired trafficking of cDCs explains the reduction of Th1 responses in BMT mice after infection with MHV-68; however, it is difficult to explain the increased pathogenic Th17 responses in the lungs of BMT mice, since similar numbers of Th17 cells were activated in the dLNs of both non-BMT and BMT mice (Figure 3B). We thus wanted to tackle this question by determining the function of each $\mathrm{CDC}$ subset in priming Th1 and Th17 cells after infection with MHV-68. Batf3 is required for the development of cDC1s, and mice deficient in Batf3 have defective $\mathrm{CD} 8^{+} \mathrm{T}$ cell responses to viral pathogens $(48,49)$. As expected, BMT mice receiving BM from Baft3 $3^{--}$mice (Baft3 $3^{--}$BMT) do not develop cDC1s after reconstitution of immune cells (Figure $5 \mathrm{~A})$. Notch2 is critical for a subset of cDC2s in the gut and spleen necessary to prime Th17 responses, but interestingly, conditional $\mathrm{KO}$ of Notch2 in CD11c-expressing cells (Notch2 ${ }^{\text {cKO }}$ ) did not reduce the numbers of $\mathrm{cDC} 2 \mathrm{~s}$ in the lung $(50,51)$. In agreement with these findings, BMT mice receiving Notch2 ${ }^{\text {cKO }}$ BM have abundant cDCs in their lungs (Figure $5 \mathrm{~A}$ ). Percentages of cDC2s in Notch2 ${ }^{\text {cKO }}$ BMT mice are similar to 
A

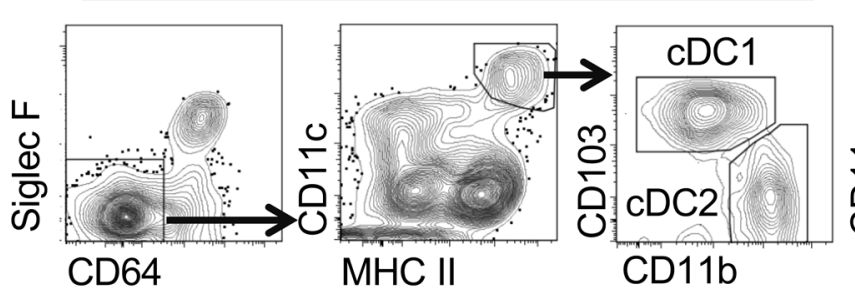

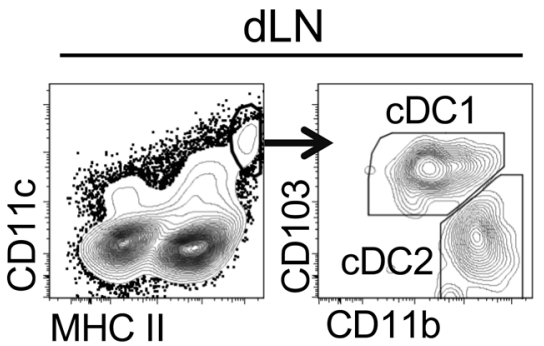

$\mathrm{MHC}$ II
B

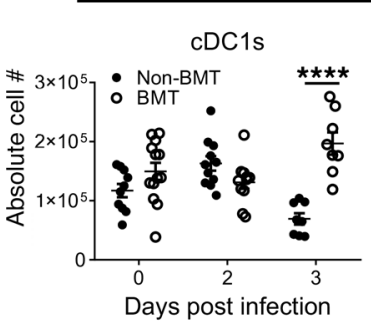

C

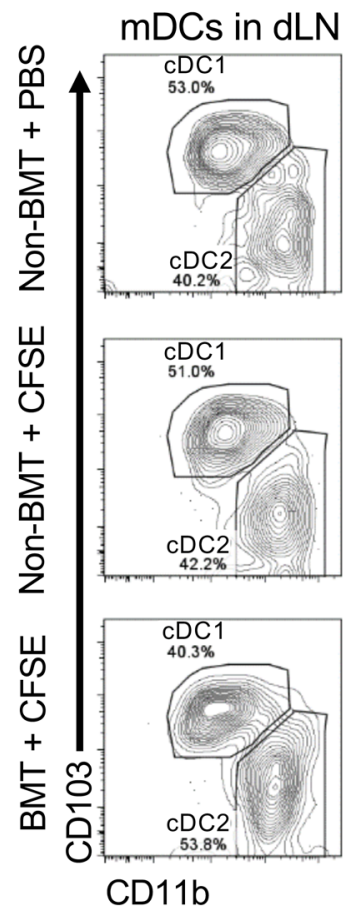

Lung

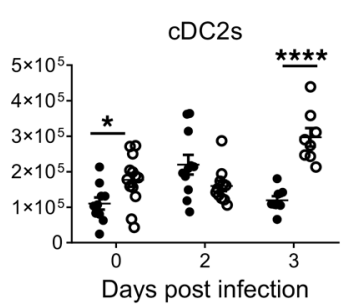

$\mathrm{dLN}$
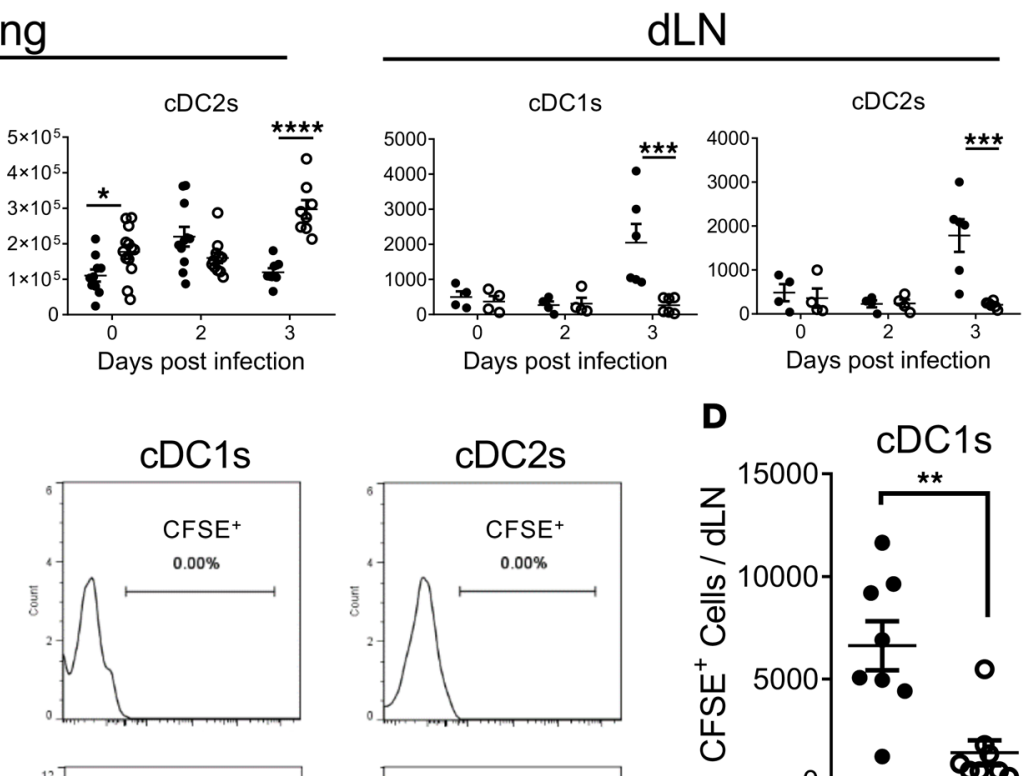

D

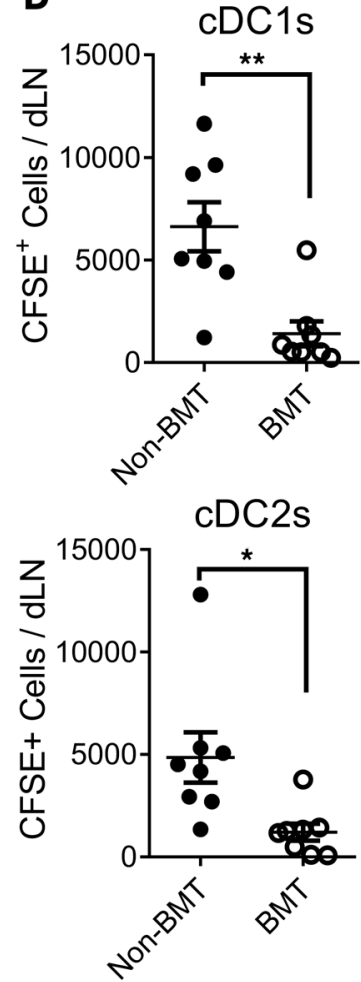

Figure 2. Impaired migration of cDCs from the lung to dLN in BMT mice determined by the kinetics of cDC subsets in the lung and dLN or tracking CFSElabeled lung cDCs. (A) Gating strategy. Lung or dLN Single-cell suspensions were prepared from mice at 3 dpi. Doublet-cell excluded live lung cells were plotted as Siglec-F vs. CD64 to exclude Siglec- $\mathrm{F}^{+}$and/or CD64+ macrophages and monocyte-derived cells. Among the remaining lung cells, cDCs were identified as a CD11 ${ }^{\text {hi }} \mathrm{MHCII}{ }^{\text {hi }}$ population, which can be further differentiated as CDC1s and CDC2s based on their expression of CD103 and CD11b. Similarly, migratory cDCs in $\mathrm{dLN}$ were identified as a CD11 $\mathrm{c}^{+} \mathrm{MHCl}{ }^{\text {hi }}$ population with $\mathrm{CDC1}$ and $\mathrm{CDC2}$ subsets. (B) Kinetics of $\mathrm{CDC1s}$ and $\mathrm{CDC2s}$ in the lungs or dLN of non-BMT or BMT mice at 0 , 2, or 3 dpi $(n=8-13$, lung; $n=4-6$, dLN). The absolute numbers of cDC1s or CDC2s per lung or dLN were calculated by multiplying the total cell number of the organ with the percentage of the cell type determined by flow cytometry (mean $\pm \mathrm{SEM}$ ). Symbols represent individual data points. ${ }^{* * *} P<0.001$; ${ }^{* * * *} P<$ 0.0001 , Student's $t$ test (2 tailed) between non-BMT and BMT mice. Similar results were obtained in 2 additional experiments. (C and D) Two days after viral infection, non-BMT or BMT mice were instilled with CFSE dye via oropharyngeal aspiration. Lung dLNs were harvested at 18 hours after CFSE instillation. Single-cell suspensions were prepared for antibody staining and flow cytometric analysis. (C) Live single dLN cells were pregated for mDCs as A, and 2-parameter contour plots for CD103 and CD11b expression were analyzed for CDC1 and CDC2 subsets. Numbers indicate the percent of cells in the gate. Single-color histograms determined the percent of CFSE ${ }^{+}$cells in each CDC subset. (D) Absolute numbers of CFSE ${ }^{+}$cells of each cDC subset per dLN of non-BMT ( $n=8$ ) or BMT $(n=8)$ mice, calculated by multiplying the total cell number of a dLN with the percentage of CFSE ${ }^{+}$cells (mean \pm SEM). Symbols represent individual mouse data points. Data were pooled from 2 independent experiments. ${ }^{*} P<0.05 ;{ }^{* *} P<0.01$, Student's $t$ test ( 2 tailed). BMT, BM transplantation; cDC1, type 1 conventional DC; dLN, draining lymph node; $\mathrm{mDC}$, migratory DC. 
A Th1 cell \#

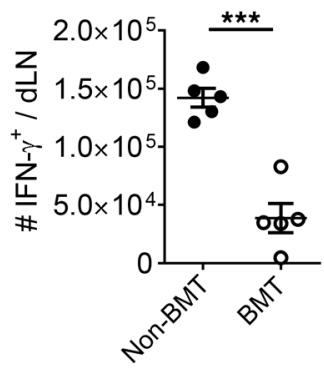

Th1\%

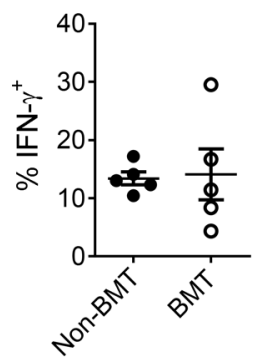

B Th17 cell \#

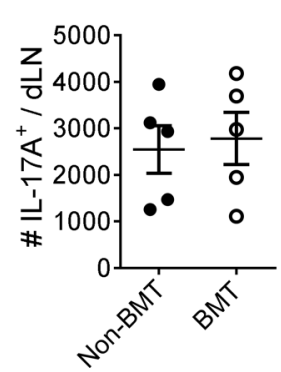

Th17\%

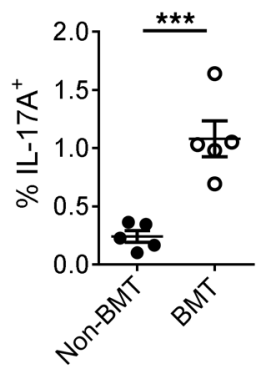

\section{C}
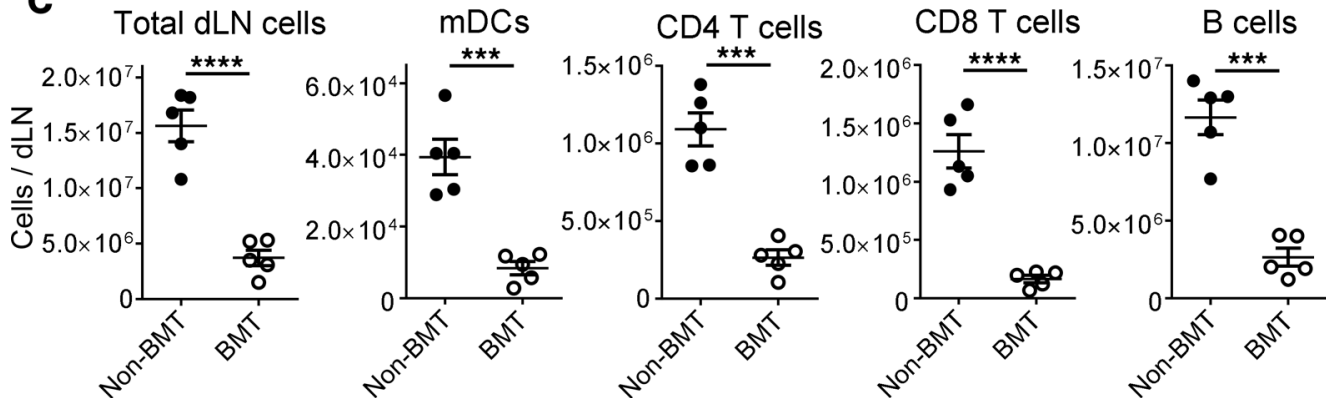

Figure 3. Th cell differentiation and cellularity in dLNs are correlated with DC migration phenotypes in non-BMT or BMT mice. (A) Absolute numbers IFN- $\gamma^{+}$Th1 cells per $\mathrm{dLN}$ and percent of Th1 cells among CD4 $4^{+} \mathrm{T}$ cells (pregated for $\left.\mathrm{CD}^{2} 5^{+} \mathrm{CD} 90.2^{+} \mathrm{CD3}^{+} \mathrm{CD} 4^{+}\right)$. (B) Absolute numbers of IL-17A+ Th17 cells per dLN and percent of Th17 cells among $\mathrm{CD}^{+}{ }^{+}$cells. (C) Total cell numbers of dLNs of non-BMT or BMT mice at $7 \mathrm{dpi}$ with MHV- 68 were determined by hemocytometer counting. The absolute numbers of $\mathrm{mDCs}, \mathrm{CD} 4^{+} \mathrm{Th}, \mathrm{CD} 8^{+} \mathrm{T}$, and $\mathrm{B}$ cells per $\mathrm{dLN}$ were determined by total dLN cell numbers and percentage of flow cytometry based on cell surface markers. Mean \pm SEM is shown for all panels and $n=5$ for each group. Similar results were obtained in 2 additional experiments. Symbols represent individual mouse data points. ${ }^{* *} P<0.001 ;{ }^{* * *} P<0.0001$, Student's $t$ test ( 2 tailed). BMT, BM transplantation; dLN, draining lymph node; mDC, migratory DC.

non-BMT mice but are slightly reduced compared with WT BMT. As expected, Notch2 ${ }^{2 K o}$ BMT mice have reduced percentages (Supplemental Figure 1A; supplemental material available online with this article; https://doi.org/10.1172/jci.insight.130210DS1) and numbers of cDC2s (Supplemental Figure 1B) in the spleen. Furthermore, Notch $2^{2 K O}$ BMT mice fail to develop splenomegaly 3 weeks after infection with MHV-68 (Supplemental Figure 1C), due to a failure in stimulating the expansion of $\mathrm{CD} 4^{+} \mathrm{T}$ cells and B cells in the spleen (Supplemental Figure 1D). Thus, unlike in the spleen, Notch2 function does not appear to be required for the development or survival of $\mathrm{cDC} 2 \mathrm{~s}$ in the lung, but the role of Notch2 in $\mathrm{cDC} 2 \mathrm{~s}$ during the priming of Th responses against lung infection is not known.

Deficiency of Batf 3 or Notch 2 in BMT mice does not affect the percentage or absolute numbers of Th1 cells in the lungs or dLNs of BMT mice at $7 \mathrm{dpi}$ (Figure 5B), suggesting that neither cDC1s nor Notch2 functionally dependent cDC2s are solely required for Th1 responses after MHV-68 infection. In contrast, Notch2 deficiency, presumably specific to cDC2s, diminishes the increase in Th17 responses to MHV-68 infection in BMT mice (Figure 5C). These data suggest that Notch2 function is required by $\mathrm{CDC} 2 \mathrm{~s}$ for increasing Th17 responses in BMT mice. Previous studies have shown that conditional deletion of IFN regulatory factor 4 (Irf4) in cDCs selectively diminishes a lung-resident $\mathrm{CD} 11 \mathrm{c}^{\text {hi }} \mathrm{CD} 11 \mathrm{~b}^{+} \mathrm{SIRPa}{ }^{+} \mathrm{CD} 24^{+}$ DC subset and causes reduction in both Th17 responses $(52)$ and $\mathrm{Th} 2$ responses $(53,54)$ in the lungs. Similarly, we found that BMT mice with IRF4 deficiency in cDCs failed to increase Th17 responses after infection with MHV-68 (Supplemental Figure 1E). Loss of cDC1s in Baft3 ${ }^{-/}$BMT mice caused further increased Th17 responses in BMT mice (Figure 5C), suggesting that the presence of $\mathrm{cDC} 1 \mathrm{~s}$ suppresses Th17 responses. Interestingly, lifting the suppressive function of Bat 3 in non-BMT mice does not increase Th17 responses (Supplemental Figure 2), presumably because CDC2s in non-BMT mice are not activated by MHV-68 infection, which is evidenced by low production levels of pro-Th17 cytokines (Figure 1C). Despite the importance of cDCs in antiviral infections $(48,49)$, deficiency in either Batf 3 or Notch2 in cDCs does not have an impact on viral lytic replication at 7 dpi (Supplemental Figure 3). Taken together, 
A
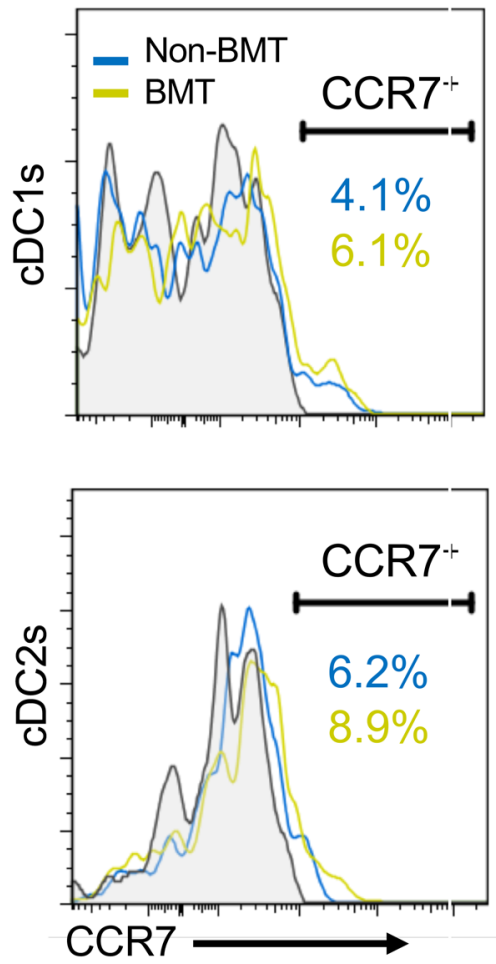

B

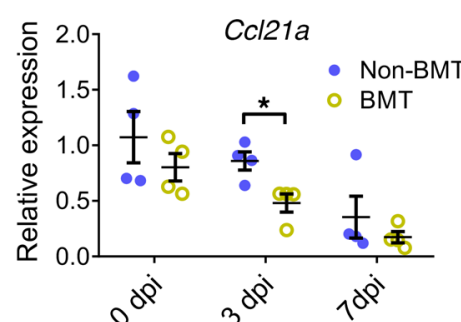

(1)

음
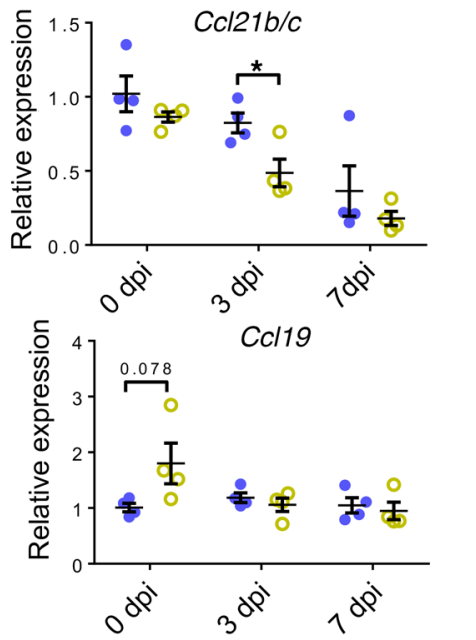

dLN
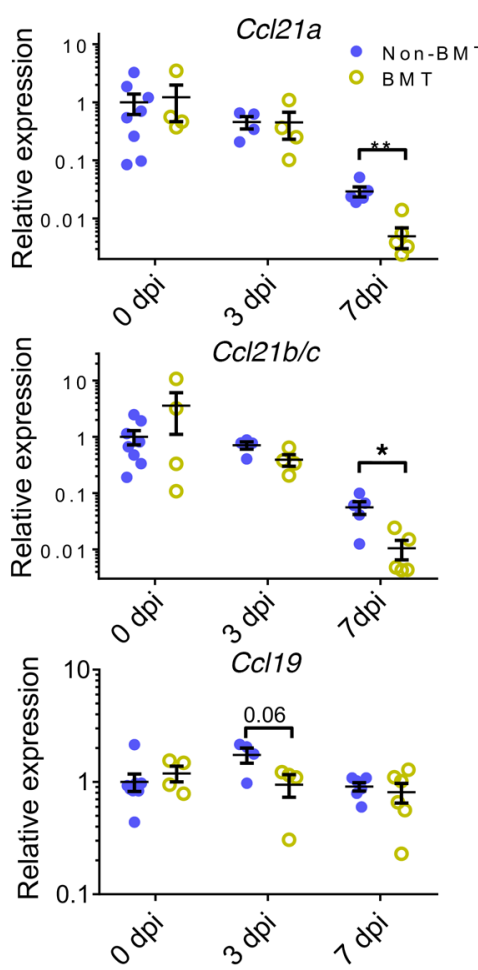

C
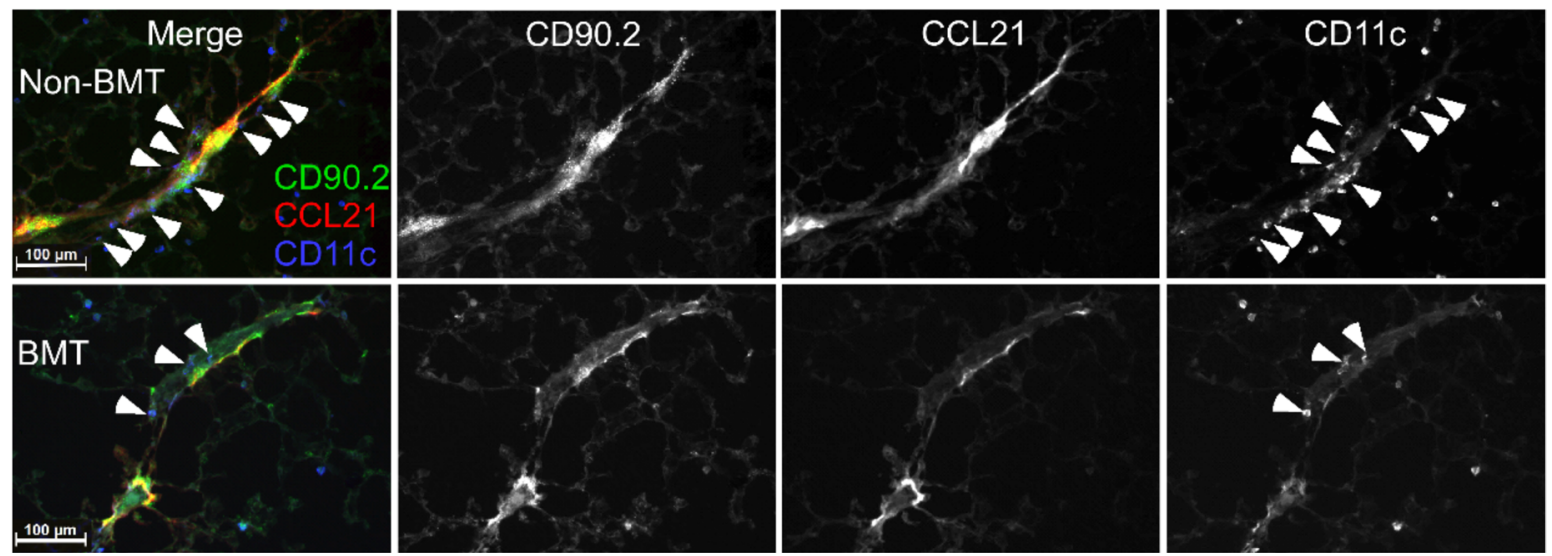

Figure 4. The expression of chemokine Ccl21 in the lung is reduced in BMT mice, but the expression of CCR7 on cDCs is not affected by BMT. (A) Representative histograms determined the percentage of CCR $7^{+}$cells in each CDC subset at 2 dpi. Gray areas represent the fluorescence minus 1 (FMO) control. Numbers indicate the percent of cells in the gate. Similar results were obtained in 2 additional experiments. (B) Relative mRNA abundance of $C c / 21 a, C c / 21 b / c$, and $C c / 19$ in dLNs and lung tissues of non-BMT or BMT mice at 0,3 , or $7 \mathrm{dpi}\left(n=4 \sim 8\right.$, Mean \pm SEM). Symbols represent individual mouse data points. ${ }^{*} P<0.05$; ${ }^{* *} P$ $<0.01$, Student's $t$ test (2 tailed). (C) Multicolor immunofluorescence staining of lung frozen sections prepared at 3 dpi. Labeling of CD90.2 protein (green) identifies lymph vessels in the lung. Labeling of CCL21 protein (red) is colocalized with CD90.2. CD11c (blue) labels DCs and alveolar macrophages. There are numerous CD11c ${ }^{+}$cells within or in contact with lymph vessels (arrowheads) in non-BMT lungs but fewer such cells in the BMT lungs. Images represent 2-3 mice per group and 2 independent experiments. Scale bar: $100 \mu \mathrm{m}$. BMT, BM transplantation; CDC1, type 1 conventional DC; dLN, draining lymph node.

our data demonstrate that Notch2 function in $\mathrm{CDC} 2 \mathrm{~s}$ is required for the increased Th17 responses, while presence of cDC1s suppresses Th17 responses in BMT mice after infection with MHV-68.

Migration of $c D C 2 s$ into lung $d L N$ is not required for Th17 responses in the lung. The migration of both cDC1s and $\mathrm{cDC} 2 \mathrm{~s}$ into the dLNs is impaired in a similar manner (Figure 2), yet the Th1 and Th17 responses in the lungs of BMT mice are differentially regulated, suggesting that $\mathrm{CDC}$ trafficking has different impacts in priming Th1 or Th17 cells. To test this possibility, we performed a BMT using BM cells from $C C R 7^{--}$donors so that, after reconstitution, the DCs and $\mathrm{T}$ cells in the recipient mice will not 
A WT non-BMT

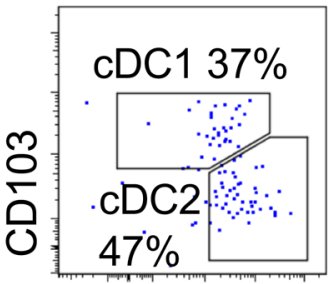

CD11b

B Th1

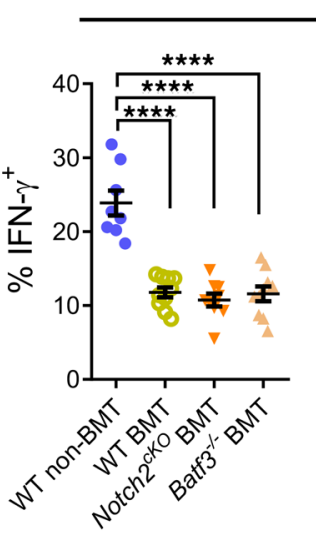

Lung

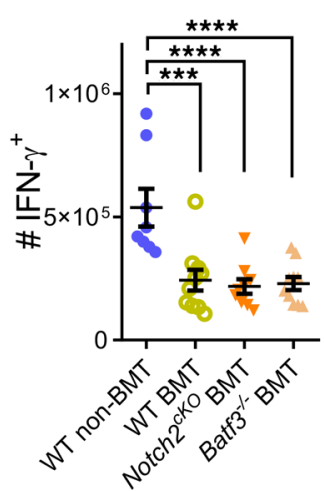

c Th17

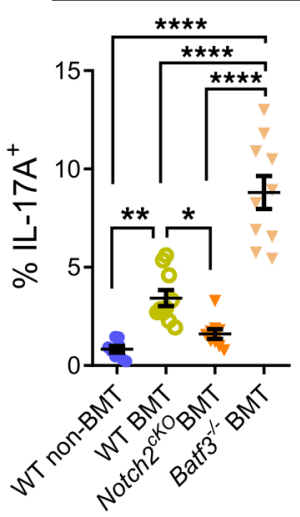

Lung

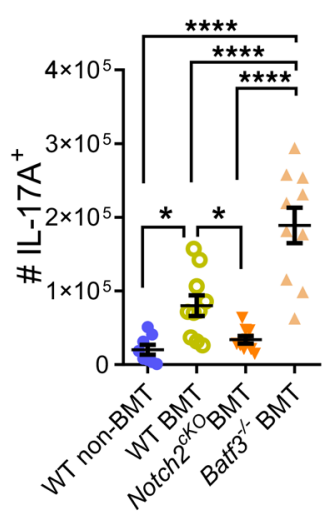

D

\section{pol}

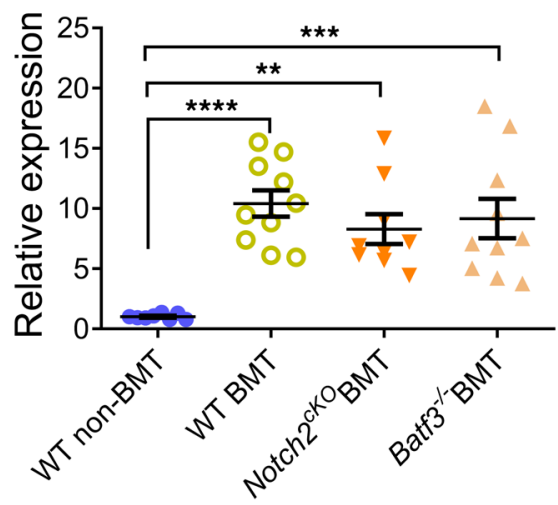

Notch2 ${ }^{\mathrm{cKO}} \mathrm{BMT}$
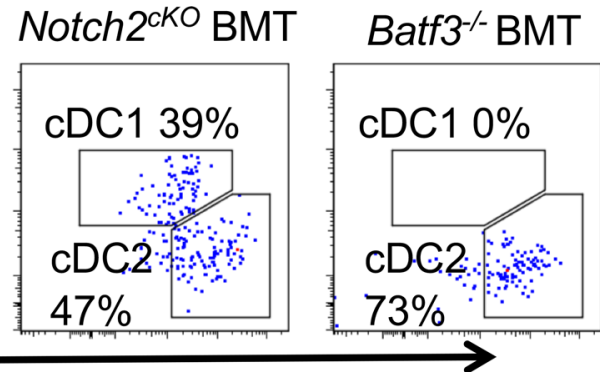

$56 \%$

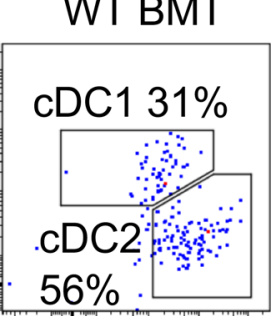

$47 \%$

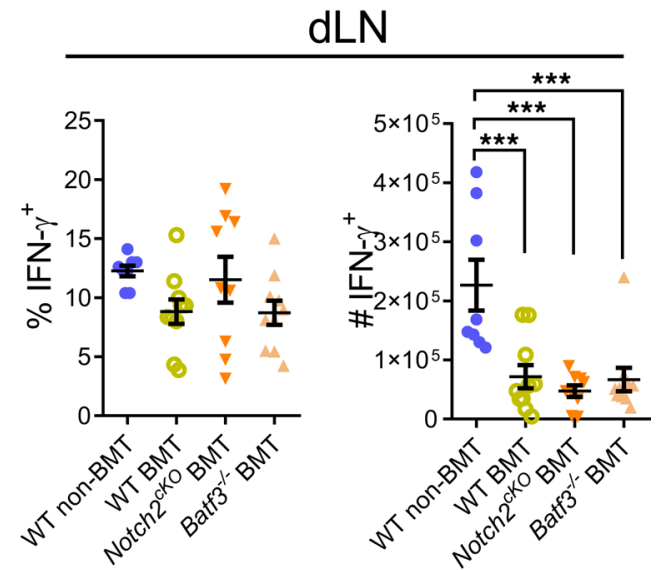

dLN

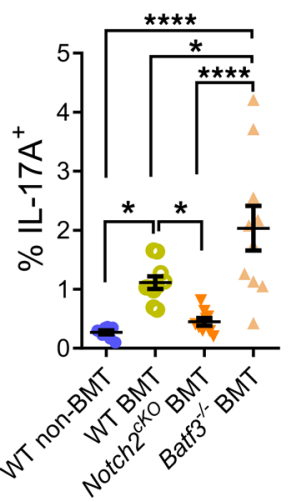

Figure 5. Notch2 function in $\mathrm{CDC2}$ is required for Th17 response, while Batf3dependent $C D C 1 s$ suppress Th17 response in BMT mice after infection with MHV-68.

(A) Representative 2-color histograms for CD103 and CD11b expression were gated for $\mathrm{CDC1}$ or $\mathrm{CDC2}$ cells in the lungs from age-matched WT non-BMT mice or BMT mice that received BM cells from WT, Notch2 ${ }^{\text {ckO }}$, or Batf3 $3^{-/-}$mice. CDCs are pregated as shown in Figure 2A. Numbers indicate the percentage of cells in the gate. (B) Percent and absolute numbers of IFN- $\gamma^{+}$ Th1 cells among $\mathrm{CD}^{+} \mathrm{T}$ cells (pregated for CD45+CD90.2+C$\mathrm{D}^{+} \mathrm{CD}^{+}$) per lung or dLN determined by flow cytometry and total cell numbers (mean \pm SEM). (C) Percent and absolute numbers of IL-17A ${ }^{+}$Th17 cells among $\mathrm{CD}^{+} \mathrm{T}$ cells (pregated for $\mathrm{CD} 45^{+} \mathrm{CD} 90.2^{+} \mathrm{CD3}^{+} \mathrm{CD}^{+}$) per lung or dLN determined by flow cytometry and total cell numbers (mean \pm SEM). (D) Relative mRNA abundance of viral $p o l$ gene or $g B$ gene in lung tissues (mean \pm SEM). In B, C, and $\mathbf{D}$, lungs and dLNs were of non-BMT $(n=8)$ or BMT mice that received BM cells from WT $(n=10), \operatorname{Notch}_{2}{ }^{\text {KOO }}(n=9)$, or Batf3 $^{-1-}(n=10)$ mice. Symbols represent individual mouse data points. Data were pooled from 2 independent experiments. ${ }^{*} P<0.05$; ${ }^{* *} P<0.01$; ${ }^{* * *} P<0.001 ;{ }^{* * *} P<0.0001$, 1-way ANOVA followed by a Tukey's multiple comparisons test. BMT, BM transplantation; CDC1, type 1 conventional DC; $\mathrm{dLN}$, draining lymph node. 
A Th1

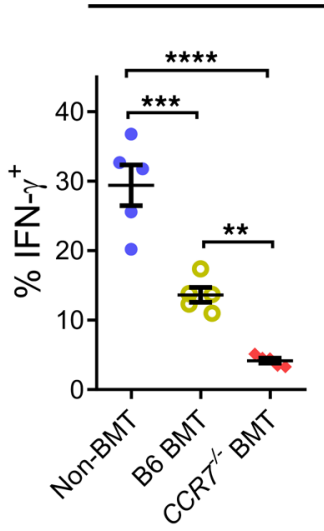

Lung

B Th17

Lung

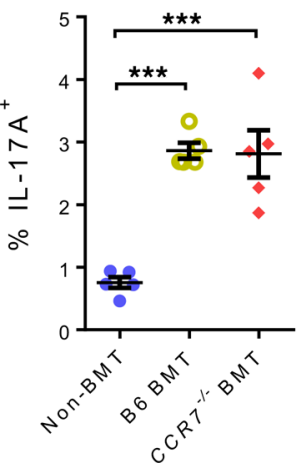

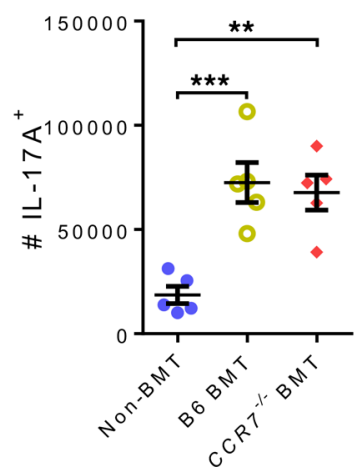
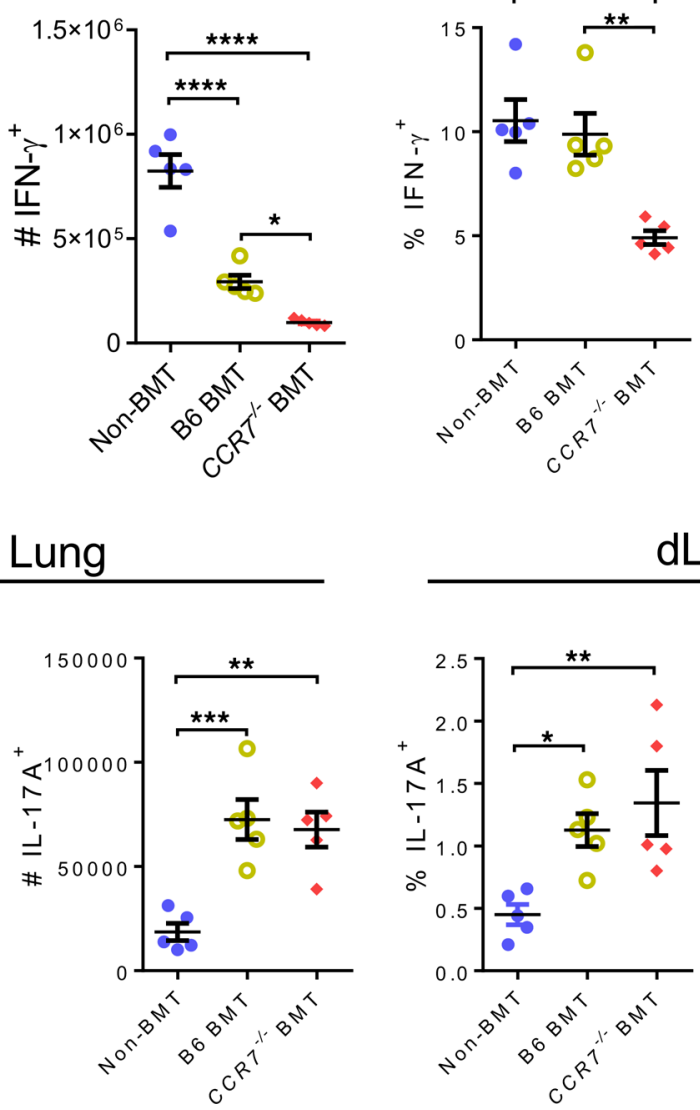

dLN

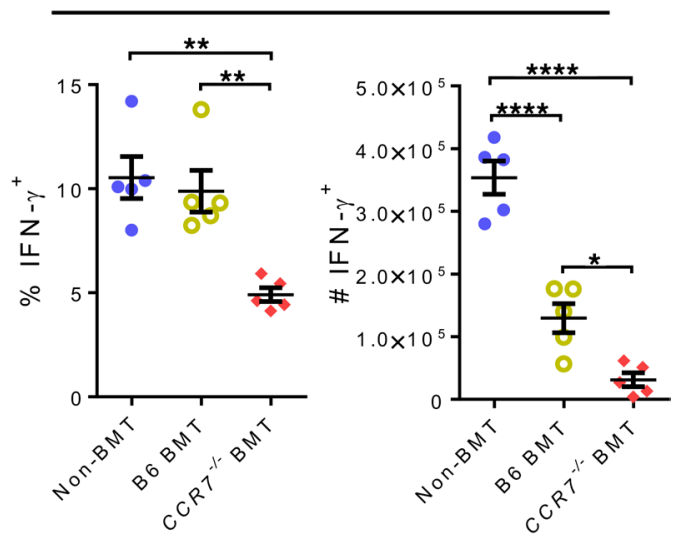

$\mathrm{dLN}$

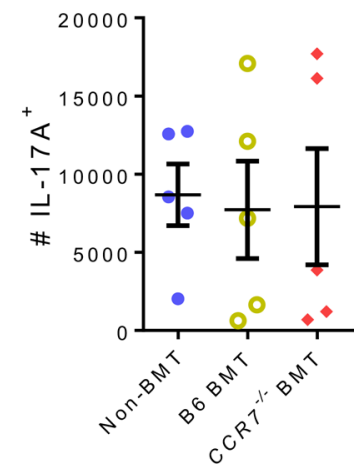

Figure 6. Migration of $\mathrm{CDCs}$ is essential for Th1 responses but not for Th17 responses. Live single lung cells prepared from non-BMT mice or BMT mice receiving B6 or CCR $7^{-1-} \mathrm{BM}(n=5$ for each group) were pregated for Th cells (CD45+C$\left.\mathrm{D3}^{+} \mathrm{CD} 90.2^{+} \mathrm{CD4} 4^{+}\right)$. (A) Percent and absolute numbers of IFN- $\gamma^{+}$Th1 cells among CD4+ Th cells per lung of B6 non-BMT,

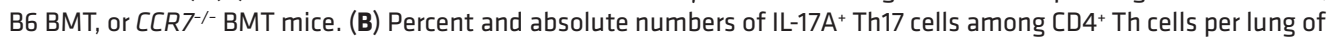
B6 non-BMT, B6 BMT, or CCR $7^{-1-}$ BMT mice. Symbols represent individual mouse data points. Mean $\pm \mathrm{SEM}$ is shown for all panels. Data are representative of 2 independent experiments. ${ }^{*} P<0.05$; ${ }^{* *} P<0.01$; ${ }^{* *} P<0.001 ;{ }^{* * *} P<0.0001$, 1 -way ANOVA followed by a Tukey's multiple comparisons test. BMT, BM transplantation; dLN, draining lymph node.

respond to CCL21. As expected, CCR $7^{--}$to WT BMT mice showed minimal Th1 responses in the lungs and dLNs at $7 \mathrm{dpi}$ (Figure 6A). Surprisingly, the percentages and numbers of Th17 cells in the lungs of $C C R 7^{-1-}$ to WT BMT mice remained as high as those of WT BMT mice (Figure 6B). Also similar to WT BMT mice, $C C R 7^{-1-}$ BMT mice have significantly higher percentages but comparable numbers of Th17 cells in their dLNs compared with non-BMT mice (Figure 6B). The sphingosine 1-phosphate (S1P) receptor agonist fingolimod (FTY720) blocks the egress of lymphocytes, so the migration of both activated and naive $\mathrm{T}$ cells to sites of inflammation is blocked about 10-12 hours after application of FTY720 (55-57). Furthermore, FTY720 increases blood-borne DCs and reduces DCs in LNs and spleen (58). Therefore, the activated Th cells found in the lungs are presumably primed locally from the naive $\mathrm{T}$ cells that arrived in the lungs before the administration of FTY720. We administered FTY720 $1 \mathrm{mg} / \mathrm{kg} /$ day via i.p. injection starting $3 \mathrm{dpi}$, and as expected, the total $\mathrm{T}$ cells and $\mathrm{CD} 4^{+} \mathrm{T}$ cells in the lung are reduced at $7 \mathrm{dpi}$ (Supplemental Figure 3, A and B). Although the absolute numbers of Th1 and Th17 cells are much lower in FTY720-treated BMT mice (Supplemental Figure 3, C and D), the percentage of Th cells remain unchanged after FTY720 treatment (Supplemental Figure 3, E and F). Thus, both data strongly suggest that $\mathrm{CDC} 2 \mathrm{~s}$ can prime Th17 responses both in the lungs and dLNs of BMT mice and CCR7-dependent migration into the dLNs is not absolutely required for $\mathrm{cDC} 2 \mathrm{~s}$ to prime Th17 responses, while $\mathrm{cDC}$ trafficking to the $\mathrm{dLN}$ is essential for priming strong Th1 responses. 


\section{Discussion}

SCT recipients are at high risk of infection even long after immune reconstitution. We have determined that impaired $\mathrm{cDC}$ trafficking is an underlying cellular mechanism responsible for the reduced protective Th1 responses to herpesviral infection by exploiting a murine syngeneic BMT model of late infection with MHV-68. The impaired cDC trafficking in BMT mice limits the opportunity for $\mathrm{CDCs}$ to prime Th cells in the dLNs. Furthermore, Notch2 function in CDC2s is required for the increased Th17 responses in BMT mice, while cDC1s can partially suppress this activity. Importantly, Th17 cells can be primed both in the lungs and dLNs, and thus allow for increased pathogenic Th17 responses even when the migration of $\mathrm{cDCs}$ to dLNs is impaired.

The interaction between CCR7 on CDCs and CCL21 protein orchestrate nearly every step of cDC migration to dLNs (reviewed in ref. 47). This interaction first guides mature $\mathrm{cDCs}$ to follow the gradient of CCL21 toward and into the lumen of lymphatic vessels in infected tissues (43). Once inside, cDCs first crawl along the vessel wall guided by the CCL21 gradient toward collecting lymphatic vessels (59) and then are passively transported by the lymph flow into LNs (60). Cells traveling within the afferent lymph are drained into the LN subcapsular sinus, where CCL21 gradients once again guide cDC migration into the LN parenchyma (61). We found that the cell surface expression of CCR7 on cDCs is not altered by BMT (Figure 4A), but the expression of Ccl21 in the lung is downregulated at 3 dpi (Figure

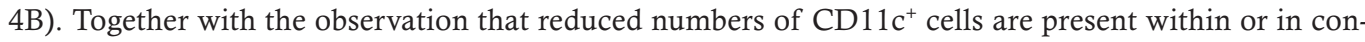
tact with lymphatic vessels in the lungs of BMT mice (Figure 4C), we propose that the initial steps of recruiting $\mathrm{cDCs}$ into lymphatic vessels in the lung are dysregulated in BMT mice. Future studies are required to understand the cellular and molecular events involved in $\mathrm{cDC}$ trafficking that are dysregulated in BMT recipient lung tissue.

It has been long recognized that the dLNs are small and disorganized in BMT recipients, and BMT mice generate poor responses to bacterial and viral infections (19-21, 62). The levels of CCL21 protein in dLN stroma have previously been reported to be reduced in BMT mice at steady-state $(19,21)$, but we did not detect such a reduction of $C c l 21$ at the transcription level (Figure 4B). A transient downregulation of chemokine production within dLNs was observed in both non-BMT and BMT dLNs at 7 dpi, which has been reported to help orchestrate local cellularity and minimize competition for space and resources in activated lymphoid tissues (63). BMT dLNs reduce their production of $C c l 21$ at 7 dpi more dramatically than non-BMT dLNs (Figure 4B). CCL21 is also essential for recruiting naive T cells into LNs (22). It is thus likely that the downregulation of $C c l 21$ after the flush of incoming $\mathrm{CDCs}$ reduces the recruitment of naive $\mathrm{T}$ cells and contributes to low cellularity of T cells, as well as B cells, in the dLNs of BMT mice.

Defects in cDC trafficking have been implicated in diseases. For example, ASAP1 protein regulates matrix degradation and cDC migration through its involvement in actin and membrane remodeling (64). Individuals with genetic variants that reduce the expression of $A S A P 1$ are associated with susceptibility to Mycobacterium tuberculosis, due to the impaired migration of $M$. tuberculosis-infected cDCs (64). In patients with chronic obstructive pulmonary disease (COPD), the functions of DCs are somewhat controversial. Several studies found that DCs accumulate in the small airways $(65,66)$ and drive NK cytotoxicity to kill lung epithelial cells (67). However, such increases in DCs in COPD small airways were not detected in another study (68).

The understanding of the division of labor among the different lung DC subsets has rapidly expanded in recent years. The cDC2 subset appears to be heterogeneous, as it supports both Th2 responses to house dust mite (41) and Th17 responses to lung fungal infection with Aspergillus fumigatus (52). Further studies found that Klf4 is required by a set of $\mathrm{cDC} 2 \mathrm{~s}$ to sustain Th2 responses to Blomia tropicalis mites (69), while Notch2 is required by cDC2s for Th17 responses in defense against Citrobacter rodentium in spleen and intestine $(50,51)$. Since Notch2 deficiency does not cause reduction of cDC2 cell numbers in the lung $(50,51)$, whether or not the function of Notch2 is required for Th17 responses in the lung was not defined. Here, we demonstrate that Notch2 is indeed required for Th17 responses to MHV-68 infection in BMT mice (Figure 5C). Surprisingly, cDC2s can prime Th17 cells both in the infected lungs and the dLNs, as evidenced in CCR $7^{-1-}$ or FTY720-treated BMT mice (Figure 5 and Supplemental Figure 3). The precise sites of interaction between $\mathrm{CDC} 2 \mathrm{~s}$ and Th cells are not yet determined. While we have not noticed well-structured tertiary lymphoid follicles in the lungs of BMT mice after infection, there is a large amount of lymphocyte infiltration that organizes in a patchy manner, which may provide unique niches for $\mathrm{cDC} 2 \mathrm{~s}$ to prime Th cells (34). 
Mouse $\mathrm{cDC} 1 \mathrm{~s}$ are required for cross-presenting antigens to $\mathrm{CD} 8^{+} \mathrm{T}$ cells for antiviral and antitumor immunity $(48,49)$. Lung cDC1s can prime both Th1 and Th17 responses in vitro $(70)$, but we found that cDC1s are not solely required for Th1 responses, and the presence of these $\mathrm{CDC} 1 \mathrm{~s}$ can suppress excessive Th17 responses in BMT mice (Figure 5, B and C). Potentially, the loss of cDC1s in Batf $3^{-1-}$ BMT mice increases the opportunities for $\mathrm{cDC} 2 \mathrm{~s}$ to interact with more naive T cells. On the other hand, the cytokines released from $\mathrm{cDC} 1 \mathrm{~s}$ may inhibit the priming of Th17 by cDC2s. IL-2 secreted by cDC1s has been reported to mediate the suppression of Th17 responses in a mouse model of invasive pulmonary aspergillosis (71). We found that lung APCs isolated from infected BMT mice express significantly lower levels of $I l 2$ after restimulation with MHV-68 ex vivo compared with normal lung APCs (Supplemental Figure 4A). When supplementing the coculture of BMT APCs and CD4 $4^{+} \mathrm{T}$ cells with $10 \mathrm{ng} / \mathrm{ml}$ of recombinant mouse IL-2, the expression of $I l 17 a$ in $\mathrm{CD}^{+} \mathrm{T}$ cells was significantly suppressed (Supplemental Figure 4B). Similarly, supplementation of the coculture of BMT APCs and CD4 ${ }^{+} \mathrm{T}$ cells with conditioned medium of non-BMT APCs also significantly suppressed the expression of 1117 a (Supplemental Figure 4B). Supplementation of IL-2 in the culture can also increase the expression of IFN- $\gamma$ (Supplemental Figure 4C). However, in vivo administration of IL-2 to BMT mice did not suppress Th17 differentiation (Supplemental Figure 4, D and E). Rather, due to the expansion of total $\mathrm{CD}^{+}$and $\mathrm{CD} 8^{+} \mathrm{T}$ cells stimulated by IL-2, the numbers of both Th17 and Th1 cells per lung were increased (Supplemental Figure 4, D-F). The administration of IL-2 also did not suppress viral replication in BMT mice (data not shown). Thus, further studies are warranted to determine how cDC1s might suppress Th17 in infected BMT mice in vivo.

Notably, the cDC2s prime Th17 responses against MHV-68 infection only in BMT mice - not in non-BMT mice. Even in Batf3 ${ }^{-/-}$non-BMT mice in which the suppression from cDC1s is lifted, Th17 response is still minimal after infection with MHV-68 (Supplemental Figure 2). These data indicate that the microenvironmental conditions in BMT mice may have dictated the ability of $\mathrm{cDC} 2 \mathrm{~s}$ to promote Th17 responses to MHV-68. The production of IL-6 (72) and IL-23 (73) is essential for cDC2s to prime Th17 cells. The APCs isolated from BMT mice produce higher levels of IL-6 and IL-23 than normal APCs when stimulated with MHV-68 (Figure 1C and ref. 24). DCs sense exogenous pathogens or tissue damage through pattern recognition receptors such as TLRs to stimulate NF- $\mathrm{BB}$ or other signaling pathways to upregulate inflammatory cytokines. The regulation of IL-6 expression and translation is fine-tuned at multiple levels (reviewed in ref. 74). MHV-68 can be recognized by TLR2 and stimulate the expression of IL-6 (75). In addition, many bacterial-, fungi-, and damage-associated molecular patterns can also bind to TLR2 or TLR9 and stimulate NF- $\mathrm{kB}$, leading to the expression of IL-6. BMT conditioning is associated with tissue damage, and we have shown that the lung microbiome community structure and diversity is significantly shifted after MHV-68 infection in BMT mice (28), which we speculate may provide suitable conditions for stimulating cDC2s to become pro-Th17 APCs.

EBV and MHV-68 can cause splenomegaly in humans and mice, respectively. Mice develop a striking splenomegaly 2-3 weeks after infection with increased numbers of $\mathrm{B}$ cells and $\mathrm{CD} 4^{+}$and $\mathrm{CD} 8^{+} \mathrm{T}$ cells (76). The development of MHV-68-induced splenomegaly depends on $\mathrm{CD}^{+} \mathrm{T}$ cells (76) and viral lytic replication in the B cells $(77,78)$. Infected DCs can pass the virus to B cells (79). We found that mice with conditional $\mathrm{KO}$ of Notch2 in CD11 $\mathrm{c}^{+}$cells do not develop splenomegaly (Supplemental Figure 1, C and D). Since lung $\mathrm{CDC} 2 \mathrm{~s}$ are required to induce $\mathrm{T}$ follicular helper cell (Tfh) differentiation in response to antigens (80) and support germinal center reactions (81), Notch2-dependent cDC2s are likely essential for both passing virus to B cells and subsequently stimulating germinal center reactions through Tfh cells. Note that MHV68 infection can cause a considerable amount of B cells to express CD11c (82); thus, Notch2 in these B cells is knocked out in our experimental system. Knock out of Notch2 in these cells may also impair their proliferation and development in a similar manner as those seen in marginal zone or follicular B cells $(83,84)$.

In conclusion, our study identifies impaired $\mathrm{cDC}$ trafficking as the underlying cellular mechanism responsible for the reduced protective Th1 responses against MHV-68 infection in BMT mice. The priming of pathogenic Th17 responses that requires Notch2 function in $\mathrm{CDC} 2 \mathrm{~s}$ can occur in the lung without cDC migration into the dLNs. Moreover, we revealed that the impaired $\mathrm{CDC}$ trafficking is not due to low expression levels of CCR7 on cDCs but is more likely due to defects in production of CCL21 in the lung, most likely in the lymphatic endothelial cells after BMT. How the BMT procedure affects the lung cell production of CCL21 or other molecules critical for $\mathrm{CDC}$ migration in response to MHV-68 infection will be the focus of future studies. Collectively, our study provides insights into the reason SCT recipients develop severe pulmonary consequences secondary to altered immune responses to viral infections. 


\section{Methods}

Mice. C57BL/6 (B6), Batf3- (B6.129S[C]-Batf3 ${ }^{\text {tm1Kmm} / J), ~ C D 11 c-C r e ~(B 6 . C g-T g[I t g a x-c r e] 1-1 R e i z / J), ~}$

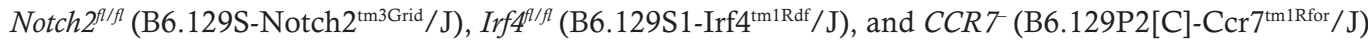
were acquired from The Jackson Laboratory. Notch $2^{f / f l}$ or Irf $4^{f / f l}$ mice were crossed to CD11c-Cre mice to generate Notch2 ${ }^{c K O}$ or Irf4 ${ }^{C K O}$ mice. All mice were maintained on C57BL/6 background in a specific pathogen-free animal facility.

Syngeneic BMT and MHV-68 infection. Recipient B6 WT mice were treated with 13 Gy total body irradiation (split dose) using a ${ }^{137} \mathrm{Cs}$ irradiator, followed by tail vein injection of $5 \times 10^{6}$ whole BM cells from WT, Batf3 ${ }^{-/-}$, Notch2 ${ }^{\text {cKO }}$, Irf4cKO ${ }^{c K O}$ or $C C R 7^{--}$mice. Inoculation of MHV-68 (VR-1465, ATCC) was carried out at the fifth week after BMT, when total numbers of hematopoietic cells were fully reconstituted in the lungs and spleen (32). Non-BMT or BMT mice were infected with $5 \times 10^{4}$ pfu of murine MHV-68 i.n. after being anesthetized with ketamine and xylazine.

Cell preparation. Mice were euthanized with $\mathrm{CO}_{2}$ (Metro Welding Supply), and the lungs were perfused with $5 \mathrm{ml}$ PBS (Invitrogen) delivered via the right ventricle of the heart before being isolated. The posterior mediastinal dLNs that the lung cDCs migrate into (85) were isolated. Single-cell suspensions of leukocytes were prepared from whole lungs by collagenase digestion for flow cytometry, as described previously (86). Briefly, each minced lung was incubated in $15 \mathrm{ml}$ of complete media with 1 $\mathrm{mg} / \mathrm{ml}$ collagenase (Boehringer Mannheim Biochemical) and $17 \mathrm{U} / \mathrm{ml}$ DNase I (MilliporeSigma) for 30 minutes at $37^{\circ} \mathrm{C}$. The digested tissue was drawn through the bore of a $10-\mathrm{ml}$ syringe repeatedly and filtered through $100-\mu \mathrm{m}$ mesh.

Flow cytometry. To characterize T lymphocytes, lung, or dLN, single cells were stimulated with PMA $(0.05 \mu \mathrm{g} / \mathrm{ml}$; MilliporeSigma) and ionomycin $(0.75 \mu \mathrm{g} / \mathrm{ml}$; MilliporeSigma) for 4 hours in the presence of GolgiStop protein transport inhibitor (BD Pharmingen) before antibody staining. An aliquot of $1 \times$ $10^{6}$ cells was first blocked by anti-CD16/CD32 (Fc block; BD Pharmingen, clone 2.4G2) antibodies and then stained with fluorochrome-conjugated antibodies on ice against cell surface markers CD45 (30-F11), CD90.2 (53-2.1), CD3 (145-2C11), CD4 (GK1.5), CD8 (53-6.7), and CD19 (1D3), all from BD Pharmingen. The cells were then permeabilized and fixed with Foxp3 staining buffer set (eBioscience) and stained for intracellular IL-17A (TC11-18H10) and IFN- $\gamma$ (XMG1.2) from BD Pharmingen following the manufacturer's instructions. For characterization of lung APCs, lung or dLN single cells were stained with antibodies on ice specific for CD45 (30-F11), CD11c (N418), CD103 (2E7), I-A b (AF6-120.1), Siglec-F (E50-2440), CD24 (M1/69), and CD68 (FA-11) from BioLegend, and CD11b (M1/70) from BD Pharmingen. Staining cell surface CCR7 were done by first incubating cells with anti-CCR7 (4B12, BioLegend) for 30 minutes at $37^{\circ} \mathrm{C}$ prior to additional surface staining on ice as above. Cells were analyzed on a LSRFortessa cell analyzer for flow cytometry (BD Biosciences).

Immunostaining of lung sections and microscopy. Immunofluorescence staining of frozen lung sections has been reported previously $(24,87)$. Briefly, frozen lung sections were fixed in $10 \%$ formalin, permeabilized by $0.1 \%$ Triton X-100 (MilliporeSigma), and followed by blocking with $1 \%$ BSA (MilliporeSigma) in PBS. The lung sections were then incubated with primary antibodies overnight at $4^{\circ} \mathrm{C}$, followed by secondary antibodies for 1 hour at room temperature. Primary antibodies and dilutions used include the following: rat anti-CD90.2/Thy-1.2 antibody (clone 30-H12, BD Biosciences Pharmingen, 1:300), goat anti-CCL21 (polyclonal, R\&D Systems, 1:100), and Armenian hamster anti-CD11c conjugated with PE/ Dazzle 594 (clone N418, BioLegend, 1:100). Secondary antibodies include donkey anti-rat IgG antibody (catalog A18740) conjugated with FITC (1:200) and donkey anti-goat IgG antibody conjugated with Alexa Fluor 647 (1:200; catalog A-21447) from Invitrogen. Images were collected on a Zeiss ApoTome fluorescent microscope using AxioVision software.

APCs and $C D 4^{+} T$ cells coculture. APCs were enriched by $\mathrm{CD} 11 \mathrm{c}^{+}$microbeads (Miltenyi Biotec) from lung single-cell suspensions of BMT or non-BMT mice at 3 dpi. $\mathrm{CD}^{+} \mathrm{T}$ cells were enriched by $\mathrm{CD} 4^{+}$ microbeads (Miltenyi Biotec) from BMT lung at 10 dpi. In a 48 -well plate, $4 \times 10^{4}$ cell/well APCs were incubated with 0.125 multiplicity of infection (MOI) of MHV- 68 for 3 hours before addition of $4 \times 10^{5} \mathrm{~T}$ cells. Cells were cultured in $0.5 \mathrm{ml}$ RPMI 1640 medium (Invitrogen) supplemented with 10\% FCS (Invitrogen), 1\% HEPES (Invitrogen), and $0.1 \% \beta$-mercaptoethanol (Invitrogen), or in conditioned medium collected from a 2-day culture of non-BMT lung APCs (4 parts of conditioned medium and 1 part of fresh medium) for 4 days. Where indicated, recombinant mouse IL-2 (PeproTech) was added to cultures to a final concentration of $10 \mathrm{ng} / \mathrm{ml}$. 
Table 1. Primers and probes for quantitative PCR

\begin{tabular}{|c|c|c|}
\hline Gene (ref.) & Oligo & Primer sequence \\
\hline \multirow[b]{2}{*}{ MHV-68 pol (86) } & Forward & 5'-ACAGCAGCTCGCCATAAAGG-3' \\
\hline & Reverse & 5'-TCCTGCCCTGGAAAGTGATG-3' \\
\hline \multirow{3}{*}{ MHV-68 gB (86) } & Forward & 5'-CGCTCATTACGGCCCAAA-3' \\
\hline & Reverse & 5'-ACCACGCCCTGGACAACTC-3' \\
\hline & Probe & 5'-TTGCCTATCACAAGCTCACCACCA-3' \\
\hline GAPDH (88) & Probe & 5'-CTCCTCGACCCTCGTCCCGT-3' \\
\hline \multirow{3}{*}{ IL-23 p19 (24) } & Forward & 5'-CTCCCTACTACGACTCAGCCAACT-3' \\
\hline & Reverse & 5'-ACTCAGGCTCGGCATCTCTT-3' \\
\hline & Probe & 5'-AGCCAGAGGATCACCCCCGGG-3' \\
\hline IL-6 (89) & Forward & 5'-GACTTCCATCCAGTTGCCTTCT-3' \\
\hline IL-12 p35 (24) & Probe & 5'-ACAACAAGAGGGAGCTGCCTGCC-3' \\
\hline \multirow{3}{*}{ Ccl21a (90) } & Forward & 5'- ATGGCTCAGATGATCACTCTGAGC - $3^{\prime}$ \\
\hline & Reverse & 5'- GTACTTAAGGCAGCAGTCCTCA-3' \\
\hline & Probe & 5'- AGCCTGGTCCTCGCTCTCTCCATCC -3' \\
\hline \multirow{3}{*}{ Cc/21b/c (90) } & Forward & 5'-AGGCAGTGATGGAGGGGGA-3' \\
\hline & Reverse & 5'-GCTTAGAGTGCTTCCGGGGTA-3' \\
\hline & Probe & 5'-TTCTTCCTTCCTATAGCCTCGGACAATACTCTAGG-3' \\
\hline \multirow{3}{*}{ Ccl19 (91) } & Forward & 5'-CTTCCGCATCATTAGCACCC -3' \\
\hline & Reverse & 5'-TCATTAGCACCCCCCAGAGT-3' \\
\hline & Probe & 5'-TGGACCTTCCCACСCCCAACTCTG-3' \\
\hline
\end{tabular}

Quantitative PCR. Total RNA was isolated from cultured cells or lung tissues by TRIzol (Invitrogen) according to manufacturer's instructions. The relative amount of mRNA for viral genes or mouse genes was assayed by real-time reverse transcription PCR (real-time RT-PCR) using thermocycler ABI Prism 7000 (Applied Biosystems Inc.) using a previously described protocol (24). Mouse GAPDH was used as an internal control gene. Gene-specific primers and probes are detailed in Table 1.

ELISA. ELISA for IFN- $\gamma$ or IL-17A was performed using R\&D Systems kits (catalogs DY 485 and DY 421, respectively) according to manufacturer's instructions.

In situ CFSE labelling. CFSE ( $5 \mu \mathrm{M}$; Invitrogen) was prepared by diluting a $50 \mu \mathrm{M}$ CFSE stock in DMSO (MilliporeSigma) with PBS and by then instilling $50 \mu 1$ into each anesthetized mouse through oropharyngeal aspiration.

FTY720 or IL-2 treatment. Mice were injected i.p. daily with $1 \mathrm{mg} / \mathrm{kg}$ FTY720 (Cayman Chemical) dissolved in sterile PBS between day 3 and 6 after infection with MHV-68. BMT mice received daily i.p. injections of recombinant murine IL-2 starting at 2 dpi with MHV-68 at a dose of 20,000 IU until 6 dpi. At 7 dpi, mice were euthanized for assays. Nontreated control mice received in equivalent volume of sterile PBS.

Statistics. When groups of 2 were compared, 2-tailed Student's $t$ tests were used to determine significance; when groups of 3 or more were compared, 1-way ANOVA was utilized with a Tukey's multiple comparisons test to determine significance.

Study approval. All murine experiments were performed according to NIH guidelines and approved by the IACUC at the University of Michigan.

\section{Author contributions}

$\mathrm{XZ}$ and BBM designed the study. CAW, MMC, PRC, and XZ performed animal studies. CAW and XZ analyzed data and wrote the initial manuscript. $\mathrm{XZ}$ and BBM revised the manuscript, and all other authors critically reviewed the manuscript drafts and approved the final version. 


\section{Acknowledgments}

This work is supported by the postdoctoral translational scholar grant MICHR 2UL1TR000433 (XZ), HHV-6 Foundation Dharam Ablashi Research Fund Grant (XZ), and NIH grants HL144481 and AI117229 (BBM). The authors thank the Unit for Laboratory Animal Medicine, the Experimental Irradiation Core, and the Flow Cytometry Core Facility funded by P30CA046592 at the University of Michigan for technical support.

Address correspondence to: Xiaofeng Zhou, Department of Internal Medicine, 4062 BSRB, 109 Zina Pitcher Place, Ann Arbor, Michigan 48109-2200, USA. Phone: 734.647.9968; Email: xiazhou@umich.edu.

1. Tomblyn M, et al. Guidelines for preventing infectious complications among hematopoietic cell transplantation recipients: a global perspective. Biol Blood Marrow Transplant. 2009;15(10):1143-1238.

2. Styczynski J, et al. Increased risk of infections and infection-related mortality in children undergoing haematopoietic stem cell transplantation compared to conventional anticancer therapy: a multicentre nationwide study. Clin Microbiol Infect. 2016;22(2):179.e1-179.e10.

3. Wingard JR, Hsu J, Hiemenz JW. Hematopoietic stem cell transplantation: an overview of infection risks and epidemiology Infect Dis Clin North Am. 2010;24(2):257-272.

4. Admiraal R, et al. Viral reactivations and associated outcomes in the context of immune reconstitution after pediatric hematopoietic cell transplantation. J Allergy Clin Immunol. 2017;140(6):1643-1650.e9.

5. Fedele R, et al. The impact of early CD4+ lymphocyte recovery on the outcome of patients who undergo allogeneic bone marrow or peripheral blood stem cell transplantation. Blood Transfus. 2012;10(2):174-180.

6. Kim DH, Sohn SK, Won DI, Lee NY, Suh JS, Lee KB. Rapid helper T-cell recovery above 200 x $106 / 1$ at 3 months correlates to successful transplant outcomes after allogeneic stem cell transplantation. Bone Marrow Transplant. 2006;37(12):1119-1128.

7. Ritter J, et al. Donor CD4 T Cell Diversity Determines Virus Reactivation in Patients After HLA-Matched Allogeneic Stem Cell Transplantation. Am J Transplant. 2015;15(8):2170-2179.

8. Storek J, Gooley T, Witherspoon RP, Sullivan KM, Storb R. Infectious morbidity in long-term survivors of allogeneic marrow transplantation is associated with low CD4 T cell counts. Am J Hematol. 1997;54(2):131-138.

9. Storek J, et al. Reconstitution of the immune system after hematopoietic stem cell transplantation in humans. Semin Immunopathol. 2008;30(4):425-437.

10. Martin PJ, et al. Life expectancy in patients surviving more than 5 years after hematopoietic cell transplantation. J Clin Oncol. 2010;28(6):1011-1016.

11. Abou-Mourad YR, et al. Long-term outcome after allo-SCT: close follow-up on a large cohort treated with myeloablative regimens. Bone Marrow Transplant. 2010;45(2):295-302.

12. Wingard JR, et al. Long-term survival and late deaths after allogeneic hematopoietic cell transplantation. J Clin Oncol. 2011;29(16):2230-2239.

13. Norkin M, et al. Characteristics of Late Fatal Infections after Allogeneic Hematopoietic Cell Transplantation. Biol Blood Marrow Transplant. 2019;25(2):362-368.

14. Jantunen $\mathrm{E}$, et al. Late non-relapse mortality among adult autologous stem cell transplant recipients: a nation-wide analysis of 1,482 patients transplanted in 1990-2003. Eur J Haematol. 2006;77(2):114-119.

15. Antin JH, Ferrara JL. Cytokine dysregulation and acute graft-versus-host disease. Blood. 1992;80(12):2964-2968.

16. Chklovskaia E, Nowbakht P, Nissen C, Gratwohl A, Bargetzi M, Wodnar-Filipowicz A. Reconstitution of dendritic and natural killer-cell subsets after allogeneic stem cell transplantation: effects of endogenous flt3 ligand. Blood. 2004;103(10):3860-3868.

17. DiCarlo J, et al. Cytokine and chemokine patterns across 100 days after hematopoietic stem cell transplantation in children. Biol Blood Marrow Transplant. 2014;20(3):361-369.

18. Henden AS, Hill GR. Cytokines in Graft-versus-Host Disease. J Immunol. 2015;194(10):4604-4612.

19. Kelly RM, et al. Short-term inhibition of p53 combined with keratinocyte growth factor improves thymic epithelial cell recovery and enhances T-cell reconstitution after murine bone marrow transplantation. Blood. 2010;115(5):1088-1097.

20. Kelly RM, et al. Keratinocyte growth factor and androgen blockade work in concert to protect against conditioning regimen-induced thymic epithelial damage and enhance T-cell reconstitution after murine bone marrow transplantation. Blood. 2008;111(12):5734-5744.

21. Stefanski HE, Jonart L, Goren E, Mulé JJ, Blazar BR. A novel approach to improve immune effector responses post transplant by restoration of CCL21 expression. PLoS ONE. 2018;13(4):e0193461.

22. Stein JV, et al. The CC chemokine thymus-derived chemotactic agent 4 (TCA-4, secondary lymphoid tissue chemokine, 6 Ckine, exodus-2) triggers lymphocyte function-associated antigen 1-mediated arrest of rolling $\mathrm{T}$ lymphocytes in peripheral lymph node high endothelial venules. J Exp Med. 2000;191(1):61-76.

23. Braun A, et al. Afferent lymph-derived T cells and DCs use different chemokine receptor CCR7-dependent routes for entry into the lymph node and intranodal migration. Nat Immunol. 2011;12(9):879-887.

24. Zhou X, et al. Bone marrow transplantation alters lung antigen-presenting cells to promote TH17 response and the development of pneumonitis and fibrosis following gammaherpesvirus infection. Mucosal Immunol. 2016;9(3):610-620.

25. Reid GE, et al. Herpesvirus Respiratory Infections in Immunocompromised Patients: Epidemiology, Management, and Outcomes. Semin Respir Crit Care Med. 2016;37(4):603-630.

26. Boeckh M, et al. Late cytomegalovirus disease and mortality in recipients of allogeneic hematopoietic stem cell transplants: importance of viral load and T-cell immunity. Blood. 2003;101(2):407-414

27. Seo S, et al. Idiopathic pneumonia syndrome after hematopoietic cell transplantation: evidence of occult infectious etiologies. Blood. 2015;125(24):3789-3797. 
28. O'Dwyer DN, et al. Lung Dysbiosis, Inflammation, and Injury in Hematopoietic Cell Transplantation. Am J Respir Crit Care Med. 2018;198(10):1312-1321

29. Versluys AB, Rossen JW, van Ewijk B, Schuurman R, Bierings MB, Boelens JJ. Strong association between respiratory viral infection early after hematopoietic stem cell transplantation and the development of life-threatening acute and chronic alloimmune lung syndromes. Biol Blood Marrow Transplant. 2010;16(6):782-791.

30. Zhou X, et al. First-Onset Herpesviral Infection and Lung Injury in Allogeneic Hematopoietic Cell Transplantation. Am J Respir Crit Care Med. 2019;200(1):63-74.

31. Auletta JJ, Devecchio JL, Ferrara JL, Heinzel FP. Distinct phases in recovery of reconstituted innate cellular-mediated immunity after murine syngeneic bone marrow transplantation. Biol Blood Marrow Transplant. 2004;10(12):834-847.

32. Hubbard LL, Ballinger MN, Wilke CA, Moore BB. Comparison of conditioning regimens for alveolar macrophage reconstitution and innate immune function post bone marrow transplant. Exp Lung Res. 2008;34(5):263-275.

33. Coomes SM, Hubbard LL, Moore BB. Impaired pulmonary immunity post-bone marrow transplant. Immunol Res. 2011;50(1):78-86

34. Coomes SM, Farmen S, Wilke CA, Laouar Y, Moore BB. Severe gammaherpesvirus-induced pneumonitis and fibrosis in syngeneic bone marrow transplant mice is related to effects of transforming growth factor- $\beta$. Am J Pathol. 2011;179(5):2382-2396.

35. Gurczynski SJ, Zhou X, Flaherty M, Wilke CA, Moore BB. Bone marrow transplant-induced alterations in Notch signaling promote pathologic Th17 responses to $\gamma$-herpesvirus infection. Mucosal Immunol. 2018;11(3):881-893.

36. Kopf M, Schneider C, Nobs SP. The development and function of lung-resident macrophages and dendritic cells. Nat Immunol. 2015;16(1):36-44.

37. Worbs T, Hammerschmidt SI, Förster R. Dendritic cell migration in health and disease. Nat Rev Immunol. 2017;17(1):30-48.

38. Guilliams M, et al. Dendritic cells, monocytes and macrophages: a unified nomenclature based on ontogeny. Nat Rev Immunol. 2014;14(8):571-578

39. Murphy TL, et al. Transcriptional Control of Dendritic Cell Development. Annu Rev Immunol. 2016;34:93-119.

40. Langlet C, et al. CD64 expression distinguishes monocyte-derived and conventional dendritic cells and reveals their distinct role during intramuscular immunization. J Immunol. 2012;188(4):1751-1760.

41. Plantinga $\mathrm{M}$, et al. Conventional and monocyte-derived CD11b(+) dendritic cells initiate and maintain T helper 2 cell-mediated immunity to house dust mite allergen. Immunity. 2013;38(2):322-335.

42. Guilliams M, et al. Alveolar macrophages develop from fetal monocytes that differentiate into long-lived cells in the first week of life via GM-CSF. J Exp Med. 2013;210(10):1977-1992.

43. Weber M, et al. Interstitial dendritic cell guidance by haptotactic chemokine gradients. Science. 2013;339(6117):328-332.

44. Lo JC, et al. Differential regulation of CCL21 in lymphoid/nonlymphoid tissues for effectively attracting T cells to peripheral tissues. J Clin Invest. 2003;112(10):1495-1505.

45. Schumann K, et al. Immobilized chemokine fields and soluble chemokine gradients cooperatively shape migration patterns of dendritic cells. Immunity. 2010;32(5):703-713.

46. Kretschmer S, Dethlefsen I, Hagner-Benes S, Marsh LM, Garn H, König P. Visualization of intrapulmonary lymph vessels in healthy and inflamed murine lung using CD90/Thy-1 as a marker. PLoS ONE. 2013;8(2):e55201.

47. Permanyer M, Bošnjak B, Förster R. Dendritic cells, T cells and lymphatics: dialogues in migration and beyond. Curr Opin Immunol. 2018;53:173-179.

48. Hildner K, et al. Batf3 deficiency reveals a critical role for CD8alpha+ dendritic cells in cytotoxic T cell immunity. Science. 2008;322(5904):1097-1100.

49. Torti N, Walton SM, Murphy KM, Oxenius A. Batf3 transcription factor-dependent DC subsets in murine CMV infection: differential impact on T-cell priming and memory inflation. Eur J Immunol. 2011;41(9):2612-2618.

50. Lewis KL, et al. Notch2 receptor signaling controls functional differentiation of dendritic cells in the spleen and intestine. Immunity. 2011;35(5):780-791.

51. Satpathy AT, et al. Notch2-dependent classical dendritic cells orchestrate intestinal immunity to attaching-and-effacing bacterial pathogens. Nat Immunol. 2013;14(9):937-948.

52. Schlitzer A, et al. IRF4 transcription factor-dependent CD11b+ dendritic cells in human and mouse control mucosal IL-17 cytokine responses. Immunity. 2013;38(5):970-983.

53. Williams JW, et al. Transcription factor IRF4 drives dendritic cells to promote Th2 differentiation. Nat Commun. 2013;4:2990.

54. Bajaña S, Turner S, Paul J, Ainsua-Enrich E, Kovats S. IRF4 and IRF8 Act in CD11c+ Cells To Regulate Terminal Differentiation of Lung Tissue Dendritic Cells. J Immunol. 2016;196(4):1666-1677.

55. Chiba K, et al. FTY720, a novel immunosuppressant, induces sequestration of circulating mature lymphocytes by acceleration of lymphocyte homing in rats. I. FTY720 selectively decreases the number of circulating mature lymphocytes by acceleration of lymphocyte homing. J Immunol. 1998;160(10):5037-5044.

56. Pham TH, et al. Lymphatic endothelial cell sphingosine kinase activity is required for lymphocyte egress and lymphatic patterning. J Exp Med. 2010;207(1):17-27.

57. Caucheteux SM, Torabi-Parizi P, Paul WE. Analysis of naïve lung CD4 T cells provides evidence of functional lung to lymph node migration. Proc Natl Acad Sci USA. 2013;110(5):1821-1826.

58. Lan YY, et al. The sphingosine-1-phosphate receptor agonist FTY720 modulates dendritic cell trafficking in vivo. Am J Transplant. 2005;5(11):2649-2659.

59. Russo E, et al. Intralymphatic CCL21 Promotes Tissue Egress of Dendritic Cells through Afferent Lymphatic Vessels. Cell Rep 2016;14(7):1723-1734

60. Tal O, et al. DC mobilization from the skin requires docking to immobilized CCL21 on lymphatic endothelium and intralymphatic crawling. J Exp Med. 2011;208(10):2141-2153.

61. Ulvmar MH, et al. The atypical chemokine receptor CCRL1 shapes functional CCL21 gradients in lymph nodes. Nat Immunol. 2014;15(7):623-630.

62. Chung J, et al. Fibroblastic niches prime T cell alloimmunity through Delta-like Notch ligands. J Clin Invest. 2017;127(4):1574-1588. 
63. Mueller SN, et al. Regulation of homeostatic chemokine expression and cell trafficking during immune responses. Science. 2007;317(5838):670-674.

64. Curtis J, et al. Susceptibility to tuberculosis is associated with variants in the ASAP1 gene encoding a regulator of dendritic cell migration. Nat Genet. 2015;47(5):523-527.

65. Vassallo R, Walters PR, Lamont J, Kottom TJ, Yi ES, Limper AH. Cigarette smoke promotes dendritic cell accumulation in COPD; a Lung Tissue Research Consortium study. Respir Res. 2010;11:45

66. Demedts IK, et al. Accumulation of dendritic cells and increased CCL20 levels in the airways of patients with chronic obstructive pulmonary disease. Am J Respir Crit Care Med. 2007;175(10):998-1005.

67. Finch DK, et al. Lung Dendritic Cells Drive Natural Killer Cytotoxicity in Chronic Obstructive Pulmonary Disease via IL-15R $\alpha$. Am J Respir Crit Care Med. 2018;198(9):1140-1150.

68. Arellano-Orden E, et al. Cigarette Smoke Decreases the Maturation of Lung Myeloid Dendritic Cells. PLoS ONE 2016;11(4):e0152737.

69. Tussiwand R, et al. Klf4 expression in conventional dendritic cells is required for T helper 2 cell responses. Immunity. 2015;42(5):916-928

70. Furuhashi K, et al. Mouse lung CD103+ and CD11bhigh dendritic cells preferentially induce distinct CD4+ T-cell responses. Am J Respir Cell Mol Biol. 2012;46(2):165-172.

71. Zelante T, et al. CD103(+) Dendritic Cells Control Th17 Cell Function in the Lung. Cell Rep. 2015;12(11):1789-1801.

72. Heink S, et al. Trans-presentation of IL-6 by dendritic cells is required for the priming of pathogenic $\mathrm{T}_{\mathrm{H}} 17$ cells. Nat Immunol. 2017;18(1):74-85.

73. Gelderblom M, et al. IL-23 (Interleukin-23)-Producing Conventional Dendritic Cells Control the Detrimental IL-17 (Interleukin-17) Response in Stroke. Stroke. 2018;49(1):155-164.

74. Tanaka T, Narazaki M, Kishimoto T. IL-6 in inflammation, immunity, and disease. Cold Spring Harb Perspect Biol. 2014;6(10):a016295

75. Michaud F, Coulombe F, Gaudreault E, Kriz J, Gosselin J. Involvement of TLR2 in recognition of acute gammaherpesvirus-68 infection. PLoS ONE. 2010;5(10):e13742

76. Usherwood EJ, Ross AJ, Allen DJ, Nash AA. Murine gammaherpesvirus-induced splenomegaly: a critical role for CD4 T cells. J Gen Virol. 1996;77(Pt 4):627-630.

77. Usherwood EJ, Stewart JP, Robertson K, Allen DJ, Nash AA. Absence of splenic latency in murine gammaherpesvirus 68-infected B cell-deficient mice. J Gen Virol. 1996;77(Pt 11):2819-2825.

78. Lawler C, de Miranda MP, May J, Wyer O, Simas JP, Stevenson PG. Gammaherpesvirus Colonization of the Spleen Requires Lytic Replication in B Cells. J Virol. 2018;92(7):e02199-17.

79. Gaspar M, et al. Murid herpesvirus-4 exploits dendritic cells to infect B cells. PLoS Pathog. 2011;7(11):e1002346.

80. Krishnaswamy JK, et al. Migratory $\mathrm{CD} 11 \mathrm{~b}^{+}$conventional dendritic cells induce $\mathrm{T}$ follicular helper cell-dependent antibody responses. Sci Immunol. 2017;2(18):eaam9169.

81. Briseño CG, et al. Notch2-dependent DC2s mediate splenic germinal center responses. Proc Natl Acad Sci USA. 2018;115(42):10726-10731.

82. Rubtsova K, Rubtsov AV, van Dyk LF, Kappler JW, Marrack P. T-box transcription factor T-bet, a key player in a unique type of B-cell activation essential for effective viral clearance. Proc Natl Acad Sci USA. 2013;110(34):E3216-E3224.

83. Thomas M, Calamito M, Srivastava B, Maillard I, Pear WS, Allman D. Notch activity synergizes with B-cell-receptor and CD40 signaling to enhance B-cell activation. Blood. 2007;109(8):3342-3350.

84. Saito T, et al. Notch2 is preferentially expressed in mature B cells and indispensable for marginal zone B lineage development. Immunity. 2003;18(5):675-685.

85. Ho AW, et al. Lung CD103+ dendritic cells efficiently transport influenza virus to the lymph node and load viral antigen onto MHC class I for presentation to CD8 T cells. J Immunol. 2011;187(11):6011-6021.

86. Coomes SM, Wilke CA, Moore TA, Moore BB. Induction of TGF-beta 1, not regulatory T cells, impairs antiviral immunity in the lung following bone marrow transplant. J Immunol. 2010;184(9):5130-5140.

87. Zhou X, Moore BB. Lung Section Staining and Microscopy. Bio Protoc. 2017;7(10):e2286.

88. Sagi Y, et al. Nitric oxide regulates synaptic transmission between spiny projection neurons. Proc Natl Acad Sci USA. 2014;111(49):17636-17641.

89. Ikeda T, et al. Effects of phorbol ester-sensitive PKC (c/nPKC) activation on the production of adiponectin in 3T3-L1 adipocytes. IUBMB Life. 2009;61(6):644-650.

90. Chen SC, et al. Ectopic expression of the murine chemokines CCL21a and CCL21b induces the formation of lymph node-like structures in pancreas, but not skin, of transgenic mice. J Immunol. 2002;168(3):1001-1008.

91. Luther SA, Tang HL, Hyman PL, Farr AG, Cyster JG. Coexpression of the chemokines ELC and SLC by T zone stromal cells and deletion of the ELC gene in the plt/plt mouse. Proc Natl Acad Sci USA. 2000;97(23):12694-12699. 